\title{
A collection of 4,741 word fragments that have unique completions with respect to 146,205 words
}

\author{
JANET M. GIBSON \\ Grinnell College, Grinnell, lowa \\ and \\ JOHN O. BROOKS III \\ Stanford University School of Medicine, Stanford, California
}

\begin{abstract}
A collection of 4,741 word fragments that have a unique completion is described. All word fragments are specified by two letters (e.g., ${ }_{-} Q_{-} U_{-}$can only be completed by the word LIQUEURS). The words completing these fragments range in length from five to nine letters. The fragments are unique with respect to a pool of 146,205 words, which helps rule out the possibility that obscure words could be used as a completion to the fragments. The collection of fragments as well as the words that complete them is available in ASCII format on computer disks or in printed form.
\end{abstract}

The use of words as stimuli in psychology experiments has resulted in words' being manipulated and distorted in virtually every imaginable way-such as in their appearance (e.g., handwritten or typed; Roediger \& Blaxton, 1987a), presentation modality (e.g., visual or auditory; Crowder, 1972), or perceptibility of presentation (e.g., blurred, Bruner \& Potter, 1964; brief, Watkins \& Gibson, 1988; or degraded, Brooks, 1987). This article focuses on a particular distortion of words-the word fragment. A fragment of a word is generated by removing several of the word's letters and replacing them with blanks. Thus, a possible fragment of the word HORIZON is $\mathrm{O}_{-} \mathrm{I}_{-} \mathrm{O}_{-}$.

Word fragments have been used as stimulus materials for decades. In some instances, the word fragment has provided a way to investigate certain hypotheses. For example, Broadbent and Broadbent (1975) used them to study the word frequency effect; fragments were also used to test the meaning change hypothesis in impression formation (Watkins \& Peynircioglu, 1984). Other research has focused on the nature of fragment completion itself: Certain lexical and semantic properties of completions affect the probability of completing fragments with targets (see, e.g., Nelson, Keelean, \& Negrao, 1989), and the gradual addition of letters to a fragment lowers the probability of successful completion (see, e.g., Peynircioglu \& Watkins, 1986).

Word fragment completion is often used as a test of memory in the absence of conscious recollection. This phenomenon, which is popularly termed implicit or indirect memory (Graf \& Schacter, 1985; Richardson-

This work was funded in part by a grant from Grinnell College to the first author. Correspondence may be addressed to either J. M. Gibson, Department of Psychology, Grinnell College, Grinnell, IA 50112-0806 or J. O. Brooks III, Department of Psychiatry and Behavioral Sciences (TD114), Stanford University School of Medicine, Stanford, CA $94305-5490$ (e-mail to either gibsonj@grinl bitnet or job@icon.palo-alto.med.va.gov).
Klavehn \& Bjork, 1988), is demonstrated when subjects show a bias or facilitation from a prior experience even though they are not asked to recollect the experience. With respect to word fragments, subjects are typically first presented with a list of words and then given a series of word fragments to complete. Facilitation resulting from the prior exposure takes the form of an increased probability of completing a fragment with the previously presented word. The word fragment completion test has played a pivotal role in theoretical approaches to implicit memory. For example, Roediger and his colleagues (e.g., Roediger \& Blaxton, 1987a, 1987b; Roediger \& Challis, 1992; Roediger, Weldon, \& Challis, 1989) have used findings with the word fragment completion test to help develop their approach of transfer-appropriate processing, and Tulving and his colleagues (e.g., Hayman \& Tulving, 1989; Tulving, Schacter, \& Stark, 1982) have used it to develop their systems approach to implicit memory.

An influential study done with word fragments was one in which Tulving et al. (1982) found evidence consistent with the notion that the probability of completion of a word's fragment is independent of whether it will be recognized in an explicit test of recognition memory. Tulving et al. also published an appendix of the 192 word fragments they used, which was intended to provide fragments that had but one completion in the English language. Many researchers have used Tulving et al.'s fragments, perhaps because finding a set of unique word fragments is very time consuming and difficult. However, the number of word fragments in Tulving et al.'s set is limited, many of the words have a low frequency of occurrence in the .English language, and some of the fragments are not unique.

To create a larger set of unique word fragments, Gibson and Watkins (1988) provided a set of 1,086 fragments and their completions' frequencies of occurrence. All the fragments in Gibson and Watkins's set were unique with 
respect to the Francis and Kučera (1982) word pool and the Puzzle Solver's Handbook. However, a limitation of Gibson and Watkins's pool was that the Francis and Kučera pool-although widely used by researchers-comprised only 50,000 words, and the Puzzle Solver's Handbook, only 18,000 . After the exclusion of words with fewer than 4 or more than 10 letters, proper nouns, and nonwords (e.g., zoooop), the pool of words with which the fragments were unique was slightly less than 30,000 . Thus, some of these fragments most likely are not unique to a larger vocabulary, nor are these fragments exhaustive of the set of possible unique fragments that may be used as stimulus materials.

\section{Description of the Word Corpus}

In an effort to provide a larger, more definitive, set of unique fragments, we based our search for unique fragments on a word pool containing 360,787 words (MobyWords, from Moby Software). This corpus was generated from a wide range of sources, such as novels, textbooks, and public-domain word pools, with the objective of obtaining an extremely large pool of words.

\section{Fragment Generation}

To create the pool of word fragments, we took all five-, six-, seven-, eight-, and nine-letter words from the corpus. The only exclusion criteria we applied were that (1) no word could be a proper noun (identified as such by capitalization) and (2) no word could contain punctuation (e.g., hyphens or apostrophes). The sizes of the resulting word sets are provided in Table 1.

We next set out to obtain words that could be uniquely specified by two-letter fragments. Note that the addition of letters to a two-letter fragment will yield a unique fragment of greater length. We created all possible two-letter fragments for each word and determined whether any other word of the same length in the corpus shared the same two letters in the same positions. Table 1 provides the numbers of unique fragments for each word length. In a few instances a word could be uniquely specified with more than one two-letter fragment (e.g., $\mathrm{E}_{-}{ }_{-} \ldots \ldots \mathrm{X}$ and $-Q_{-} \ldots-X$ are unique fragments for the word EQUINOX). In these instances, we retained both fragments. An example of a fragment of each word length is provided in Table 2 .

When unique fragments are selected from a word pool as large as the one we used, there is a tradeoff between ensuring uniqueness of the fragments and frequency of

Table 1

Number of Words and Fragments According to Length

\begin{tabular}{ccc}
\hline $\begin{array}{c}\text { Word Length } \\
\text { (in Letters) }\end{array}$ & $\begin{array}{c}\text { Number } \\
\text { of Words }\end{array}$ & $\begin{array}{c}\text { Number of } \\
\text { Unique Fragments }\end{array}$ \\
\hline Five & 11,763 & 597 \\
Six & 23,543 & 684 \\
Seven & 32,044 & 858 \\
Eight & 39,109 & 1,190 \\
Nine & 39,746 & 1,412 \\
\hline
\end{tabular}

Table 2

\begin{tabular}{ccl}
\multicolumn{3}{c}{ Examples of Unique Two-Letter Word Fragments } \\
\hline $\begin{array}{c}\text { Word Length } \\
\text { (in Letters) }\end{array}$ & \multicolumn{1}{c}{ Unique Fragment } & \multicolumn{1}{c}{ Solution } \\
\hline Five & - & ENJOY \\
Six & - & CLIMAX \\
Seven & - & BOUQUET \\
Eight & D$_{-}$ & DETOXIFY \\
Nine & - & FLASHBULB \\
\hline
\end{tabular}

occurrence of the completions in the English language. Thus, a number of the unique fragments have completions that have a low frequency of occurrence. Moreover, we did not edit the corpus, and, consequently, our pool includes some words that may properly be described as obscure or may be found only in unabridged dictionaries. We found this alternative preferable to imposing what would necessarily be arbitrary standards of deletion.

\section{Hardware and Software Requirements}

The word fragments may be read by any word processor or programming language. There are no specific requirements regarding equipment specifications.

\section{Availability}

The words and the positions of the two letters that specify a unique fragment are provided in the Appendix. The Appendix is arranged alphabetically within word length, beginning with the five-letter words and ending with the nine-letter words. The two numbers that appear next to each word designate the positions of the letters that specify a unique word fragment. Because some words may give rise to more than one fragment, they are listed more than once.

The word fragments are also distributed in a text, or ASCII, file that contains four pieces of information for each fragment: (1) the word that completes the fragment; (2) the fragment itself, in which underscores have replaced the other letters; (3) the position of the first letter of the fragment; and (4) the position of the second letter of the fragment. The fragment pool is sorted in three different ways: (1) by length and then alphabetically; (2) by length and then by fragment; (3) by length and then by fragment position and fragment. The ASCII files are delimited with tabs.

The word files can be provided on 3.5-in. disks formatted for a Macintosh or an IBM-PC-compatible or in printed form by request. A copy of the collection may be obtained by sending $\$ 5$ to either author. Alternatively, a copy may be obtained through electronic mail to either author (note that some electronic mail systems may not permit transmission of the entire pool).

\section{REFERENCES}

Broadbent, D. E., \& Broadbent, M. H. P. (1975). Some further data concerning the word frequency effect. Journal of Experimental Psychology: General, 104, 297-308.

Brooks, J. O., III. (1987). Enhancing and degrading visual stimuli. Behavior Research Methods, Instruments, \& Computers, 19, 260-269. 
Bruner, J. S., \& Potter, M. C. (1964). Interference in visual recognition. Science, 144, 424-425.

Crowder, R. G. (1972). Visual and auditory memory. In J. F. Kavanagh \& I. G. Mattingly (Eds.), Language by ear and by eye: The relationships between speech and reading (pp. 251-275). Cambridge, MA: MIT Press.

Francis, W. N., \& KuCERA, H. (1982). Frequency analysis of English usage: Lexicon and grammar. Boston: Houghton Mifflin.

Gibson, J. M., \& Watkins, M. J. (1988). A pool of 1,086 words with unique two-letter fragments. Behavior Research Methods, Instruments, \& Computers, 20, 390-397.

GRAF, P., \& SCHACTER, D. L. (1985). Implicit and explicit memory for new associations in normal and amnesic subjects. Journal of $E x$ perimental Psychology: Learning, Memory, \& Cognition, 11, 501-518.

Hayman, C. A. G., \& Tulving, E. (1989). Contingent dissociation between recognition and fragment completion: The method of triangulation. Journal of Experimental Psychology: Learning, Memory, \& Cognition, 15, 228-240.

Nelson, D. L., KeElEan, P. D., \& Negrao, M. (1989). Word-fragment cuing: The lexical search hypothesis. Journal of Experimental Psychology: Learning, Memory, \& Cognition, 15, 388-397.

Peynircioglu, Z. F., \& Watkins, M. J. (1986). Cue depreciation: When word fragment completion is undermined by prior exposure to lesser fragments. Journal of Experimental Psychology: Learning, Memory, \& Cognition, 12, 426-431.

Richardson-Klavehn, A., \& BJork, R. A. (1988). Measures of memory. Annual Review of Psychology, 39, 475-543.
Roediger, H. L., III, Blaxton, T. A. (1987a). Effects of varying modality, surface features, and retention interval on priming in wordfragment completion. Memory \& Cognition, 15, 379-388.

Roediger, H. L., III, \& Blaxton, T. A. (1987b). Retrieval modes produce dissociations in memory for surface information. In D. Gorfein \& R. R. Hoffman (Eds.), Memory and learning: The Ebbinghaus Centennial Conference (pp. 349-379). Hillsdale, NJ: Erlbaum.

Roediger, H, L., III, \& Challis, B. H. (1992). Effects of exact repetition and conceptual repetition on free recall and primed wordfragment completion. Journal of Experimental Psychology: Learning, Memory, \& Cognition, 18, 3-14.

Roediger, H. L., III, Weldon, M. S., \& Challis, B. H. (1989). Explaining dissociations between implicit and explicit measures of retention: A processing account. In H. L. Roediger \& F. I. M. Craik (Eds.), Varieties of memory and consciousness: Essays in honor of Endel Tulving (pp. 3-14). Hillsdale, NJ: Erlbaum.

Tulving, E., Schacter, D. L., \& Stark, H. A. (1982). Priming effects in word-fragment completion are independent of recognition memory. Journal of Experimental Psychology: Learning, Memory, \& Cognition, 8, 336-342.

WatKINS, M. J., \& GibSON, J. M. (1988). On the relation between recognition memory and perceptual priming. Journal of Experimental Psychology: Learning, Memory, \& Cognition, 14, 477-483.

Watkins, M. J., \& Peynircioglu, Z. F. (1984). Determining perceived meaning during impression formation: Another look at the meaning change hypothesis. Journal of Personality \& Social Psychology, 46, 1005-1016. 


\section{APPENDIX \\ Words with Unique Two-Letter Fragments}

Fragment uniqueness is defined with respect to 146,205 words contained in the MobyWords corpus. Words are printed alphabetically by word length. Unique fragments can be created by providing the letters located in the positions indicated by the numbers following each word. For example, $\mathrm{AA}_{-} \mathrm{A}_{-}$uniquely cues AALII.

\section{S-letter words}

\begin{tabular}{|c|c|c|c|c|c|c|c|c|c|c|c|}
\hline aalii & 1,2 & agoho & 2,4 & avows & 2,4 & cnida & 1,2 & ekaha & 2,4 & foppy & 1,3 \\
\hline abaff & 2,5 & agsam & 2,3 & awave & 2,4 & coaxy & 1,4 & elsin & 2,3 & fpsps & 1,2 \\
\hline abamp & 2,5 & ајаја & 1,4 & awber & 2,3 & coaxy & 2,4 & enjoy & 1,3 & fremd & 4,5 \\
\hline abhor & 2,3 & ајаја & 2,4 & awful & 2,3 & cohog & 3,5 & enjoy & 3,5 & fubsy & 3,4 \\
\hline abkar & 2,3 & ajari & 2,5 & awful & 2,4 & comdg & 4,5 & envoi & 3,5 & fultz & 2,5 \\
\hline ablow & 2,5 & ajhar & 2,3 & awiwi & 2,4 & comdr & 4,5 & enzym & 1,3 & fulwa & 3,4 \\
\hline abnet & 2,3 & ajhar & 2,5 & awkly & 2,3 & comfy & 3,4 & eosin & 1,2 & fytte & 1,2 \\
\hline abord & 2,5 & akebi & 2,4 & axiom & 2,5 & contd & 4,5 & epoxy & 1,4 & ganev & 1,5 \\
\hline about & 2,4 & akeki & 2,4 & ayuyu & 2,3 & contg & 4,5 & epoxy & 2,4 & ganev & 2,5 \\
\hline abuzz & 2,5 & aknee & 2,3 & azido & 2,5 & corpn & 4,5 & equal & 2,5 & ganev & 4,5 \\
\hline abwab & 2,3 & akpek & 2,3 & azlon & 2,3 & cresc & 4,5 & equip & 2,5 & gawby & 3,4 \\
\hline abwab & 2,5 & alhet & 2,3 & azlon & 2,4 & crwth & 2,3 & equiv & 1,5 & gazoz & 1,5 \\
\hline acapu & 2,5 & alpax & 2,5 & azlon & 2,5 & ctene & 1,2 & equiv & 2,5 & gazoz & 3,5 \\
\hline acerb & 3,5 & alway & 2,3 & azoch & 2,4 & cymba & 2,4 & equiv & 3,5 & gizmo & 3,4 \\
\hline acmic & 2,3 & amalg & 4,5 & azofy & 2,4 & czars & 1,2 & esrog & 2,3 & going & 1,5 \\
\hline adage & 2,4 & amapa & 2,4 & azoic & 2,4 & czars & 2,5 & estab & 2,5 & groow & 1,5 \\
\hline addda & 2,4 & ameba & 2,4 & azoic & 2,5 & decyl & 3,4 & ethyl & 3,4 & gryde & 3,4 \\
\hline addnl & 4,5 & amelu & 2,5 & azoxy & 2,4 & demob & 3,5 & etuve & 1,4 & gteau & 1,2 \\
\hline adfix & 2,3 & amhar & 2,3 & azyme & 2,3 & depoh & 4,5 & evade & 2,4 & gtcau & 2,5 \\
\hline adieu & 2,5 & amove & 2,4 & azyme & 2,4 & deriv & 1,5 & evoke & 2,4 & hacek & 3,5 \\
\hline adjag & 2,3 & ampyx & 2,4 & babka & 3,4 & deriv & 2,5 & ewder & 2,3 & hajib & 3,5 \\
\hline adjag & 2,5 & angst & 3,4 & badju & 4,5 & dhikr & 4,5 & ewhow & 2,5 & hajji & 3,4 \\
\hline admov & 2,5 & antiq & 1,5 & batik & 3,5 & dioxy & 2,4 & exact & 2,4 & hanap & 1,5 \\
\hline adnex & 2,3 & antiq & 2,5 & beaux & 3,5 & dixit & 3,5 & excud & 2,4 & hawok & 3,5 \\
\hline adobe & 2,4 & antiq & 3,5 & befop & 3,5 & djinn & 2,5 & exdie & 2,3 & hdqrs & 1,2 \\
\hline adoxy & 2,4 & antiq & 4,5 & begob & 3,5 & djins & 2,5 & exlex & 2,5 & hdqrs & 1,3 \\
\hline adoze & 2,4 & aorta & 1,2 & bejel & 3,5 & doubt & 4,5 & expel & 2,3 & hdqrs & 2,3 \\
\hline adpao & 2,3 & apnea & 2,3 & benab & 3,5 & duhat & 1,3 & exsec & 2,3 & hdqrs & 3,5 \\
\hline adsum & 2,3 & aquae & 2,5 & benjy & 2,4 & dujan & 1,3 & exsec & 2,5 & hexyl & 1,4 \\
\hline adunc & 2,5 & archd & 4,5 & benjy & 4,5 & dvigu & 1,2 & extra & 2,5 & hexyl & 3,4 \\
\hline adusk & 2,5 & arjun & 2,3 & bezzi & 3,5 & dvigu & 1,5 & exurb & 2,5 & howff & 3,4 \\
\hline aface & 2,4 & asdic & 2,3 & bonav & 1,5 & dvigu & 2,4 & faqir & 3,4 & howff & 3,5 \\
\hline afara & 2,5 & asgmt & 2,3 & bonav & 2,5 & ecize & 2,4 & faqir & 3,5 & howso & 3,5 \\
\hline afear & 2,3 & asgmt & 2,4 & bovld & 3,4 & edify & 1,4 & fardh & 4,5 & hsien & 1,2 \\
\hline affix & 2,5 & askew & 3,5 & braxy & 1,4 & edify & 2,4 & faulx & 1,5 & hynde & 2,4 \\
\hline afire & 2,3 & askip & 3,5 & btise & 1,2 & effet & 1,2 & fhrer & 1,2 & iambi & 1,2 \\
\hline aflow & 2,5 & asoak & 2,5 & buffi & 4,5 & egypt & 2,3 & fifth & 3,5 & iambi & 1,4 \\
\hline afoam & 2,5 & asoka & 2,4 & busby & 3,4 & eject & 2,4 & finew & 1,5 & ibota & 1,2 \\
\hline afoul & 2,4 & aswim & 3,5 & bwana & 1,2 & eject & 2,5 & fjeld & 2,4 & iddhi & 2,4 \\
\hline agasp & 2,5 & ataxy & 2,4 & bwana & 2,5 & ejido & 2,4 & fjord & 2,3 & idgah & 2,5 \\
\hline agaze & 2,4 & avahi & 2,4 & byway & 2,3 & ejido & 2,5 & flamb & 1,5 & idgah & 3,5 \\
\hline agile & 2,4 & avgas & 2,3 & cabda & 3,4 & ejusd & 2,3 & fldxt & 3,4 & idose & 1,4 \\
\hline aging & 2,5 & avick & 2,5 & chivw & 4,5 & ejusd & 2,4 & fluyt & 4,5 & idyll & 1,3 \\
\hline agoge & 2,4 & aview & 2,5 & choux & 3,5 & ejusd & 4,5 & foehn & 3,4 & ifrit & 1,2 \\
\hline
\end{tabular}


5-letter words (continued)

\begin{tabular}{|c|c|c|c|c|c|c|c|c|c|c|c|}
\hline ihram & 1,2 & kylix & 1,5 & oftly & 2,4 & queer & 1,5 & strix & 2,5 & usque & 2,3 \\
\hline ijmaa & 1,2 & lanaz & 1,5 & ogeed & 2,5 & quegh & 1,4 & struv & 2,5 & utsuk & 2,3 \\
\hline ijmaa & 2,3 & lawzy & 1,4 & ogham & 2,3 & queme & 1,4 & stupp & 4,5 & uvver & 1,3 \\
\hline immov & 2,5 & lawzy & 3,4 & ogmic & 2,3 & queys & 1,4 & subch & 3,4 & uvver & 2,3 \\
\hline inaja & 1,4 & layup & 3,4 & ohmic & 2,3 & quipo & 1,5 & svelt & 1,2 & uzara & 1,2 \\
\hline inaja & 2,4 & layup & 3,5 & oicks & 1,4 & quipu & 1,5 & sways & 2,4 & verby & 1,4 \\
\hline incog & 3,5 & lesiy & 4,5 & okrug & 2,3 & qursh & 1,3 & Swizz & 2,4 & verek & 1,5 \\
\hline indef & 1,5 & lesya & 1,4 & ollav & 1,5 & rajah & 3,5 & swizz & 2,5 & vifda & 3,4 \\
\hline indef & 3,5 & livre & 3,4 & ollav & 2,5 & recpt & 3,4 & sylva & 2,4 & vijao & 3,5 \\
\hline indiv & 3,5 & lulab & 1,5 & opelu & 1,5 & rexen & 1,3 & tacso & 3,4 & vimpa & 1,4 \\
\hline induc & 4,5 & lulav & 1,5 & otkon & 2,3 & rhamn & 4,5 & tchai & 1,2 & vizzy & 1,4 \\
\hline insep & 1,5 & lulav & 2,5 & ouija & 1,4 & ritzy & 1,4 & tejon & 1,3 & vocab & 1,5 \\
\hline inwit & 1,3 & major & 3,5 & outby & 3,4 & roque & 1,3 & telex & 1,5 & vocab & 3,5 \\
\hline iroko & 1,4 & makuk & 3,5 & outdo & 3,4 & sabzi & 3,4 & thatn & 4,5 & vodka & 1,4 \\
\hline itcze & 1,4 & mamry & 3,4 & ovism & 2,5 & sajou & 1,3 & theow & 3,5 & vodka & 3,4 \\
\hline itcze & 2,4 & maniu & 4,5 & owght & 2,3 & samaj & 1,5 & thruv & 1,5 & voraz & 1,5 \\
\hline itcze & 3,4 & marys & 1,4 & owght & 2,4 & samaj & 2,5 & thruv & 2,5 & vying & 1,5 \\
\hline ixtle & 1,2 & mazic & 3,5 & oxbow & 2,5 & samaj & 3,5 & thuja & 1,4 & wahoo & 1,3 \\
\hline izard & 2,5 & milha & 3,4 & oxeye & 2,4 & samaj & 4,5 & thulr & 4,5 & wakif & 3,5 \\
\hline izing & 2,5 & misdo & 3,4 & oxfly & 2,3 & schav & 2,5 & tlaco & 1,2 & wawah & 1,3 \\
\hline iztle & 2,3 & misgo & 3,4 & oxime & 2,4 & schav & 3,5 & tmema & 1,2 & widow & 1,5 \\
\hline izzat & 1,3 & mixup & 3,5 & oxlip & 2,5 & schiz & 2,5 & tmema & 2,4 & windz & 4,5 \\
\hline izzat & 2,3 & mneme & 1,2 & oylet & 1,2 & schuh & 4,5 & topaz & 3,5 & wryly & 1,3 \\
\hline izzat & 2,4 & mohwa & 1,4 & ozena & 2,3 & scoke & 2,4 & topsl & 4,5 & xebec & 1,3 \\
\hline izzat & 2,5 & mohwa & 2,4 & pauxi & 2,4 & senvy & 3,4 & tsubo & 2,5 & xerox & 1,5 \\
\hline jarvy & 1,4 & motif & 3,5 & pauxi & 4,5 & sequa & 1,3 & twixt & 1,4 & xerus & 1,4 \\
\hline jazzy & 1,4 & mowch & 3,4 & pawaw & 3,5 & sfoot & 1,2 & twixt & 2,4 & xerus & 1,5 \\
\hline jerez & 1,5 & mowha & 3,4 & peuhl & 4,5 & shahi & 2,4 & twixt & 3,4 & xoana & 1,2 \\
\hline jiffy & 1,4 & mphps & 3,4 & pfund & 1,2 & shoyu & 3,5 & uayeb & 1,3 & xoana & 1,3 \\
\hline jinja & 1,4 & muang & 1,5 & pfund & 2,3 & sixmo & 3,4 & uayeb & 3,5 & xoana & 1,4 \\
\hline jiqui & 1,3 & mucic & 3,5 & pfund & 2,4 & sixth & 3,5 & uckia & 1,2 & xurel & 1,2 \\
\hline jixie & 1,3 & muhly & 3,4 & pfund & 2,5 & skice & 2,4 & uinal & 1,2 & xylan & 1,4 . \\
\hline jnana & 1,2 & mujik & 3,5 & piing & 2,3 & skids & 2,4 & ukase & 1,2 & xylem & 1,5 \\
\hline jough & 1,5 & myrrh & 4,5 & pilaf & 2,5 & skoot & 2,4 & unapt & 1,4 & xyrid & 1,5 \\
\hline judex & 1,5 & namaz & 3,5 & pique & 1,3 & slojd & 2,4 & unfew & 3,5 & xysti & 1,3 \\
\hline jundy & 1,4 & nanmu & 3,4 & pizza & 1,3 & slojd & 4,5 & ungag & 3,5 & xysti & 1,4 \\
\hline kahau & 3,5 & nasab & 3,5 & plupf & 4,5 & smift & 2,4 & ungka & 1,4 & xysti & 1,5 \\
\hline kazoo & 1,3 & naumk & 4,5 & pneum & 1,2 & soulz & 4,5 & ungka & 3,4 & yabbi & 1,5 \\
\hline khvat & 2,3 & navig & 1,5 & pohna & 3,4 & spahi & 2,4 & unhex & 3,5 & yacht & 1,4 \\
\hline kilij & 1,5 & navig & 3,5 & polyp & 4,5 & spave & 2,4 & unify & 1,4 & yadim & 1,5 \\
\hline kilij & 2,5 & neffy & 1,4 & ponja & 1,4 & spiff & 2,4 & unjam & 3,5 & yards & 1,4 \\
\hline kilij & 3,5 & netop & 1,5 & preux & 2,5 & spitz & 2,5 & unown & 1,4 & yclad & 1,2 \\
\hline kilij & 4,5 & ngwee & 2,3 & preux & 3,5 & sprew & 2,5 & unshy & 2,4 & yerga & 1,4 \\
\hline kiswa & 3,4 & njave & 1,2 & prexy & 3,4 & spyer & 2,3 & unzip & 3,5 & yezzy & 1,3 \\
\hline kiwis & 1,3 & noyau & 1,3 & propr & 4,5 & squam & 2,5 & upaya & 2,4 & ygapo & 1,2 \\
\hline kmole & 1,2 & nudzh & 4,5 & psych & 2,4 & squaw & 2,5 & upfly & 2,3 & ympet & 1,2 \\
\hline knezi & 2,4 & nuque & 1,3 & psych & 2,5 & squaw & 3,5 & upget & 2,3 & yojan & 1,3 \\
\hline knyaz & 3,5 & obeys & 2,4 & qiyas & 1,3 & squeg & 2,5 & upjet & 2,3 & youff & 1,4 \\
\hline kopje & 3,4 & objet & 1,3 & quads & 1,4 & squin & 2,5 & upmix & 2,3 & youff & 1,5 \\
\hline korec & 1,5 & objet & 2,3 & qualm & 1,5 & squiz & 2,5 & urubu & 1,4 & young & 1,5 \\
\hline kotow & 1,5 & ocque & 1,3 & quasi & 1,5 & sruti & 1,2 & urucu & 4,5 & youve & 1,4 \\
\hline kotow & 3,5 & ocque & 2,3 & quave & 1,4 & stacc & 4,5 & usage & 1,4 & yummy & 1,4 \\
\hline krivu & 4,5 & ocyte & 2,3 & quawk & 1,4 & starw & 4,5 & usage & 2,4 & zebub & 1,5 \\
\hline kyack & 2,5 & often & 2,5 & qubba & 1,4 & stowp & 4,5 & usque & 1,3 & zesty & 1,3 \\
\hline
\end{tabular}


5-letter words (continued)

$\begin{array}{llllllllll}\text { ziara } & 1,3 & \text { ziffs } & 1,4 & \text { zippy } & 1,4 & \text { zolle } & 1,4 & \text { zudda } & 1,3 \\ \text { ziffs } & 1,3 & \text { zihar } & 1,3 & \text { zloty } & 1,2 & \text { zowie } & 1,3 & & \end{array}$

6-letter words

\begin{tabular}{|c|c|c|c|c|c|c|c|c|c|c|c|}
\hline abbrev & 2,6 & ajoint & 2,4 & avoyer & 2,4 & climax & 2,6 & eggcup & 2,6 & galyac & 4,6 \\
\hline abbrev & 3,6 & ajoint & 2,6 & awanyu & 2,5 & cloque & 3,4 & eggcup & 3,4 & galyak & 4,6 \\
\hline abbrev & 4,6 & ajoure & 2,4 & awmous & 2,3 & colloq & 1,6 & egghot & 2,4 & ginkgo & 4,5 \\
\hline abbrev & 5,6 & ajoure & 2,5 & azilut & 2,5 & colloq & 2,6 & ejecta & 2,4 & gizmos & 3,4 \\
\hline abcess & 2,3 & ajowan & 2,4 & azimin & 2,4 & colloq & 3,6 & ejecta & 2,5 & goodby & 4,5 \\
\hline abhors & 2,3 & ajowan & 2,6 & aziola & 2,4 & colloq & 4,6 & ejidos & 2,5 & gorraf & 1,6 \\
\hline ablaze & 3,5 & akasha & 2,4 & aziola & 2,5 & colloq & 5,6 & ektene & 2,3 & granma & 4,5 \\
\hline abmhos & 2,3 & akasha & 2,5 & azonal & 2,6 & colyba & 4,5 & elcaja & 1,5 & grivna & 4,5 \\
\hline abmhos & 2,4 & akazga & 2,4 & azteca & 2,3 & colyum & 4,5 & elcaja & 3,5 & groszy & \\
\hline bsorb & 2,6 & akazga & 4,5 & azteca & 2,5 & colyum & 4,6 & elixir & 1,4 & gthite & \\
\hline absume & 2,5 & akcheh & 2,3 & azured & 2,3 & constr & 5,6 & embrew & 2,6 & gthite & 1,3 \\
\hline abwatt & 2,3 & akcheh & 2,4 & azygos & 2,3 & corojo & 4,5 & emceed & 2,3 & gyttja & 1,5 \\
\hline acajou & 2,4 & akoasm & 2,6 & azygos & 2,4 & corymb & 4,6 & empexa & 3,5 & gyttja & 4,5 \\
\hline acajou & 4,6 & alfaje & 3,5 & bagwyn & 4,5 & coryza & 4,5 & enjamb & 3,6 & hafnyl & 3,5 \\
\hline ichkan & 2,4 & alkoxy & 3,5 & bajree & 3,4 & courap & 3,6 & enjoys & 3,5 & halerz & 5,6 \\
\hline achkan & 3,4 & alkyne & 3,4 & bejape & 3,5 & couxio & 4,6 & entrez & 2,6 & hametz & 3,6 \\
\hline acmite & 2,3 & amhran & 2,3 & bejazz & 3,5 & cowpox & 3,6 & entrez & 3,6 & helluo & 5,6 \\
\hline acnode & 2,3 & amixia & 2,4 & bejazz & 3,6 & crapwa & 4,5 & epizoa & 1,4 & hexdra & 3,4 \\
\hline icquit & 2,3 & amorph & 2,5 & bejazz & 5,6 & ctenii & 1,2 & equoid & 2,6 & hexyne & 3,4 \\
\hline ddarme & 2,5 & amoved & 2,4 & benzyl & 4,5 & cwierc & 1,2 & ersatz & 3,6 & howitz & 3,6 \\
\hline idempt & 2,5 & amphib & 2,6 & bijoux & 3,6 & cwierc & 5,6 & esopgi & 4,5 & hpital & 1,2 \\
\hline idieux & 2,6 & amsath & 2,3 & bijoux & 4,6 & czechs & 2,4 & eucgia & 3,4 & ibexes & 2,4 \\
\hline adjiga & 3,5 & amuyon & 2,4 & bimeby & 3,5 & czechs & 2,5 & euvrou & 1,6 & idesia & 2,4 \\
\hline admove & 2,5 & analav & 2,6 & bisext & 2,5 & davyne & 3,4 & evejar & 1,4 & iliahi & 1,5 \\
\hline adrift & 2,5 & analav & 3,6 & bisext & 3,5 & defade & 3,5 & eveque & 2,4 & impawn & 2,5 \\
\hline idroop & 2,6 & analav & 4,6 & bkbndr & 1,2 & defuze & 3,5 & evermo & 2,5 & imperf & 2,6 \\
\hline fetal & 2,4 & naqua & 1,4 & bkbndr & 2,3 & deixis & 2,4 & evicke & 2,5 & infeft & 3,5 \\
\hline fffixt & 2,5 & anaqua & 4,6 & bkbndr & 5,6 & deject & 1,3 & evviva & 2,3 & inkpot & 3,4 \\
\hline fghan & 2,3 & antjar & 2,4 & blowby & 4,5 & deluxe & 1,5 & evzone & 2,3 & inower & 1,4 \\
\hline fghan & 2,4 & apercu & 2,6 & bonnaz & 3,6 & depths & 3,5 & exarch & 2,6 & interj & 1,6 \\
\hline field & 2,3 & aquake & 2,5 & brique & 2,4 & diiamb & 3,6 & excyst & 2,4 & interj & 2,6 \\
\hline Ifrown & 2,5 & asbest & 2,3 & bungfu & 4,5 & dimwit & 3,4 & exequy & 2,4 & interj & 3,6 \\
\hline Iftaba & 2,5 & ascitb & 2,6 & bunyip & 4,6 & djebel & 2,4 & exodoi & 2,6 & interj & 4,6 \\
\hline Igazed & 2,4 & ascitb & 5,6 & byhand & 2,3 & djehad & 2,4 & exopod & 2,4 & interj & 5,6 \\
\hline agedly & 2,4 & ashcan & 3,4 & cabuja & 3,5 & djehad & 2,6 & faquir & 1,3 & iodoxy & 2,5 \\
\hline agible & 2,4 & asquat & 2,3 & cabuja & 4,5 & djelfa & 2,5 & fidawi & 1,5 & isodef & 3,6 \\
\hline agneau & 2,6 & assize & 2,5 & cachou & 3,6 & djersa & 2,5 & fipple & 1,3 & itzebu & 2,3 \\
\hline gogic & 2,4 & ssyth & 2,4 & cajava & 3,5 & djinny & 2,6 & fixage & 3,5 & itzebu & 2,6 \\
\hline grege & 2,5 & syth & 3,4 & calxes & 2,4 & downby & 4,5 & fixups & 3,5 & itzebu & 3,6 \\
\hline rope & 2,5 & atazir & 2,4 & calxes & 3,4 & dreamt & 5,6 & fizgig & 3,4 & iurant & 1,2 \\
\hline tuaji & 2,5 & atbash & 2,3 & candyh & 5,6 & druxey & 4,6 & fjords & 1,2 & iwaiwa & 2,5 \\
\hline uaji & 3,5 & veen & 2,3 & canjac & 4,6 & duyker & 3,4 & flyoff & 1,6 & izafat & 2,4 \\
\hline ujon & 2,4 & wixt & 2,5 & ivdia & 3,4 & dvaita & 1,2 & flyoff & 2,6 & izzard & 2,3 \\
\hline yria & 2,3 & wixt & 3,5 & caxiri & 3,6 & ecbole & 2,3 & forgab & 1,6 & izzard & 2,4 \\
\hline choo & 2,3 & avanyu & 2,6 & chihfu & 4,5 & eczema & 2,3 & freijo & 2,5 & izzard & 2,5 \\
\hline enjo & 2,5 & aveloz & 2,6 & chintz & 2,6 & effigy & 2,5 & fsiest & 1,2 & jajman & 3,4 \\
\hline enjo & 2,6 & avichi & 2,6 & chogak & 4,6 & effuso & 2,6 & fuhrer & 1,3 & jajman & 3,5 \\
\hline imez & 2,6 & avijja & 2,5 & chuhra & 3,4 & egesta & 2,4 & fylgja & 4,5 & jayvee & 3,4 \\
\hline ajimez & 4,6 & avijja & 4,5 & chuzwi & 4,5 & eggcup & 2,4 & galaxy & 1,5 & jharal & 1,2 \\
\hline
\end{tabular}


6-letter words (continued)

\begin{tabular}{|c|c|c|c|c|c|c|c|c|c|c|c|}
\hline jharal & 1,3 & majoon & 3,5 & opaque & 1,4 & puszta & 3,4 & scrike & 2,5 & taysmm & 5,6 \\
\hline jigsaw & 1,6 & mangwe & 1,5 & opaque & 2,4 & puszta & 4,5 & secpar & 3,4 & tejano & 1,3 \\
\hline jimjam & 1,4 & maquis & 1,3 & orfray & 2,3 & pyjama & 2,3 & sejour & 3,6 & terfez & 4,6 \\
\hline jinsha & 1,5 & markaz & 1,6 & ormolu & 1,6 & pyrobi & 2,5 & sequel & 3,6 & thitka & 4,5 \\
\hline jinxed & 1,4 & massig & 4,6 & outawe & 1,5 & pyruwl & 2,5 & sexpot & 3,4 & thixle & 1,4 \\
\hline jobade & 1,5 & maxixe & 3,5 & outvie & 3,4 & pyuria & 2,3 & shadow & 2,6 & thujyl & 4,5 \\
\hline jodelr & 5,6 & mazuca & 3,5 & ouyezd & 5,6 & qasida & 1,3 & shafii & 4,6 & tinaja & 2,5 \\
\hline kakkak & 4,6 & mbeuer & 1,2 & overby & 2,5 & qiviut & 1,3 & shapka & 4,5 & tinaja & 3,5 \\
\hline kanaff & 1,5 & meisje & 2,5 & ovibos & 2,4 & quahog & 1,4 & shohji & 1,5 & tizwin & 3,4 \\
\hline kanyaw & 4,6 & messrs & 4,5 & oviger & 2,4 & quahog & 1,6 & shohji & 4,5 & tjaele & 2,4 \\
\hline kashga & 4,5 & mezcal & 3,4 & oxalyl & 2,5 & quayed & 1,4 & shwebo & 3,6 & tjaele & 2,5 \\
\hline khalif & 4,6 & mezuza & 1,5 & oxazin & 1,4 & queeve & 1,5 & sixgun & 3,4 & tmesis & 1,2 \\
\hline kharaj & 1,6 & mezuza & 3,5 & oxazin & 2,4 & quibus & 1,4 & sjomil & 1,2 & torque & 1,4 \\
\hline kharaj & 2,6 & miljee & 3,4 & oxford & 2,3 & quiffs & 1,4 & skelic & 2,6 & towzie & 3,4 \\
\hline kharwa & 4,5 & minxes & 1,4 & oxgang & 2,6 & quinic & 1,6 & skryer & 2,4 & toxify & 3,5 \\
\hline khodja & 1,5 & mizrah & 3,4 & oxhoft & 2,5 & quinyl & 1,5 & skycap & 2,4 & tranfd & 4,5 \\
\hline khodja & 4,5 & moshav & 1,6 & oxlike & 1,5 & quippu & 1,6 & slepez & 4,6 & tranfd & 5,6 \\
\hline khutba & 4,5 & moshav & 2,6 & oxreim & 2,3 & quisby & 1,5 & smaltz & 2,6 & transf & 2,6 \\
\hline kibitz & 2,6 & moshav & 3,6 & oxskin & 1,4 & rabfak & 4,6 & snazzy & 2,4 & transf & 4,6 \\
\hline kibitz & 3,6 & moshav & 4,6 & oxskin & 2,4 & radiov & 1,6 & solfge & 4,5 & treasr & 5,6 \\
\hline kidvid & 3,4 & muckna & 4,5 & oxyazo & 2,5 & radiov & 2,6 & soueef & 3,6 & twazzy & 2,4 \\
\hline kishke & 4,5 & muruxi & 5,6 & oxyazo & 2,6 & radiov & 3,6 & sphinx & 3,6 & tyauve & 2,5 \\
\hline klaxon & 1,4 & muscow & 4,6 & oxyopy & 3,5 & radiov & 4,6 & squabs & 2,5 & typify & 2,5 \\
\hline kodogu & 5,6 & muumuu & 2,3 & ozoner & 2,6 & radiov & 5,6 & squeel & 2,5 & typika & 2,5 \\
\hline kolhoz & 4,6 & muumuu & 5,6 & pailow & 3,6 & rahdar & 3,4 & squirr & 2,6 & tzetse & 2,5 \\
\hline kompow & 3,6 & muvule & 3,4 & pakeha & 4,5 & rakija & 1,5 & squoze & 2,5 & tzetze & 2,5 \\
\hline konfyt & 4,5 & muzhik & 3,4 & paletz & 1,6 & rakija & 3,5 & squshy & 2,4 & uakari & 1,2 \\
\hline kordax & 1,6 & muzjik & 3,4 & panisc & 5,6 & ramjet & 1,4 & squshy & 2,5 & ubique & 2,4 \\
\hline korova & 1,5 & myitis & 2,3 & pareja & 1,5 & rbound & 1,2 & squush & 2,4 & ubussu & 5,6 \\
\hline korzec & 4,6 & myowun & 2,4 & parfum & 4,6 & reavow & 4,6 & sradha & 1,2 & udaler & 1,2 \\
\hline kozuka & 3,5 & myself & 2,6 & pegbox & 3,6 & rebuff & 3,6 & staplf & 4,6 & ugsome & 2,3 \\
\hline kthibh & 1,2 & nahoor & 1,3 & peroxy & 1,5 & redupl & 3,5 & staxis & 1,4 & umfaan & 2,3 \\
\hline kthibh & 5,6 & naique & 1,4 & pfunde & 1,2 & requiz & 1,6 & subjee & 3,4 & unisex & 1,6 \\
\hline kuvasz & 3,6 & nazify & 3,5 & pfunde & 2,3 & requiz & 3,6 & subpar & 3,4 & unisex & 4,6 \\
\hline kuvasz & 5,6 & neetup & 1,6 & pfunde & 2,4 & revamp & 3,5 & subvii & 3,4 & unwoof & 3,6 \\
\hline kvutza & 2,5 & nontax & 1,6 & pfunde & 2,5 & revamp & 3,6 & suivez & 4,6 & unzone & 1,3 \\
\hline lablab & 1,6 & norkyn & 4,5 & phulwa & 4,5 & review & 3,6 & svaraj & 2,6 & upgape & 2,5 \\
\hline lachsa & 4,5 & novcic & 3,4 & pinjra & 4,5 & review & 4,6 & swaggi & 2,6 & upgape & 3,5 \\
\hline lambda & 4,5 & nritta & 1,2 & pinxit & 4,6 & rexine & 1,3 & swallo & 2,6 & upgaze & 2,5 \\
\hline langka & 1,5 & nyanza & 1,5 & piqure & 3,5 & rfound & 1,2 & swaraj & 2,6 & upjerk & 2,3 \\
\hline lapdog & 3,4 & nyanza & 2,5 & piqure & 3,6 & rizzom & 3,6 & swiwet & 2,4 & uppbad & 2,4 \\
\hline laquei & 3,6 & nylgau & 2,6 & piupiu & 5,6 & rosbif & 4,6 & swythe & 2,3 & uppbad & 3,4 \\
\hline larynx & 4,6 & oakweb & 3,4 & plewgh & 4,5 & roucou & 4,6 & syllab & 2,6 & uppuff & 2,6 \\
\hline lasque & 1,4 & oakweb & 3,6 & plexus & 4,5 & rwound & 1,2 & syzygy & 3,5 & urazin & 1,4 \\
\hline lavabo & 3,5 & obeyeo & 4,6 & popjoy & 3,4 & sarlyk & 5,6 & tafwiz & 3,4 & urheen & 2,3 \\
\hline lechwe & 1,5 & obfusk & 2,3 & prajna & 4,5 & sawbwa & 3,5 & tafwiz & 4,6 & urtext & 2,5 \\
\hline logjam & 1,4 & oboval & 2,4 & prankt & 5,6 & sawbwa & 4,5 & tanjib & 4,6 & uruisg & 5,6 \\
\hline logjam & 3,4 & offcut & 3,4 & prefab & 1,6 & schanz & 5,6 & taqlid & 3,4 & useful & 2,4 \\
\hline lujula & 1,3 & offing & 2,6 & prefab & 2,6 & schouw & 3,6 & tariqa & 1,5 & utmost & 2,3 \\
\hline lulavs & 5,6 & offset & 2,4 & pronpl & 4,5 & schouw & 4,6 & tariqa & 2,5 & uxoris & 1,2 \\
\hline lyfkie & 2,3 & ohmage & 1,2 & psovie & 2,4 & schouw & 5,6 & tariqa & 3,5 & vacouf & 1,6 \\
\hline lynxes & 1,4 & okroog & 2,4 & psywar & 2,4 & scogie & 2,4 & tariqa & 4,5 & valewe & 1,5 \\
\hline lynxes & 2,4 & okruzi & 2,5 & pufftn & 5,6 & scowls & 2,4 & tariqa & 5,6 & vedika & 1,5 \\
\hline mahzor & 3,4 & ollamh & 5,6 & pulque & 2,4 & scrawm & 5,6 & tasajo & 3,5 & vertep & 1,6 \\
\hline
\end{tabular}


6-letter words (continued)

$\begin{array}{llllllllllll}\text { viddui } & 1,6 & \text { wohlac } & \mathbf{1 , 6} & \text { xylene } & 1,4 & \text { yaqona } & 3,4 & \text { ynambu } & 1,6 & \text { zaqqum } & 4,6 \\ \text { vivify } & 3,5 & \text { wokowi } & 3,5 & \text { xylene } & 1,5 & \text { yaqona } & 3,5 & \text { yokage } & 1,5 & \text { zehner } & 1,3 \\ \text { vizsla } & 3,4 & \text { wowser } & 1,3 & \text { xyloid } & 1,5 & \text { yaxche } & 1,3 & \text { yttric } & 1,6 & \text { zigzag } & 3,4 \\ \text { volupt } & 1,5 & \text { xarque } & 1,2 & \text { xyloid } & 1,6 & \text { yaxche } & 3,5 & \text { zakkeu } & 1,3 & \text { zindiq } & 1,6 \\ \text { wahwah } & 3,4 & \text { xarque } & 1,4 & \text { xylose } & 1,5 & \text { yclept } & 1,2 & \text { zakkeu } & 1,6 & \text { zindiq } & 2,6 \\ \text { walrus } & 3,4 & \text { xeriff } & 1,5 & \text { xyloyl } & 1,5 & \text { yelver } & 1,4 & \text { zanjon } & 1,4 & \text { zindiq } & 3,6 \\ \text { warmup } & 4,6 & \text { xeriff } & 1,6 & \text { xyster } & 1,5 & \text { yetzer } & 1,4 & \text { zapupe } & 1,5 & \text { zindiq } & 4,6 \\ \text { wedeln } & 5,6 & \text { xoanon } & 1,2 & \text { xyster } & 1,6 & \text { yizkor } & 3,4 & \text { zaqqum } & 1,4 & \text { zindiq } & 5,6 \\ \text { wheyey } & 1,4 & \text { xoanon } & 1,3 & \text { yakalo } & 1,6 & \text { yliche } & 1,2 & \text { zaqqum } & 3,4 & \text { zlotys } & 1,2 \\ \text { witjar } & 1,4 & \text { xoanon } & 1,4 & \text { yanqui } & 1,4 & \text { ymmote } & 1,2 & \text { zaqqum } & 3,5 & \text { zounds } & 1,5 \\ \text { wlench } & 1,2 & \text { xylate } & 1,4 & \text { yaqona } & 1,3 & \text { ynambu } & 1,5 & \text { zaqqum } & 3,6 & \text { zoysia } & 1,3\end{array}$

\section{7-letter words}

\begin{tabular}{|c|c|c|c|c|c|c|c|c|c|c|}
\hline abacaxi & 2,6 & aeronef & 5,7 & alfaqui & 3,5 & asphyxy & 3,6 & benzoyl & 4,6 & centrev \\
\hline abacaxi & 4,6 & aerotow & 4,7 & alferez & 5,7 & asphyxy & 5,6 & bespawl & 4,6 & centrev \\
\hline abacaxi & 6,7 & affably & 2,7 & alforja & 2,6 & asqueal & 3,7 & besquib & 4,7 & centrev \\
\hline badejo & 2,6 & affloof & 2,7 & alforja & 3,6 & asseize & 2,6 & etwixt & 4,6 & halque \\
\hline badejo & 4,6 & affreux & 2,7 & alforja & 5,6 & astilbe & 5,6 & birlinn & 6,7 & hamoix \\
\hline bashed & 2,5 & affreux & 3,7 & Ihenna & 2,3 & ataraxy & 2,6 & bissext & 3,6 & chanoyu \\
\hline bfarad & 2,3 & affreux & 5,7 & liipoe & 3,4 & ataxite & 2,4 & blijver & 2,4 & chlnatri \\
\hline bhenry & 2,3 & affying & 2,4 & aljamia & 2,3 & athbash & 3,4 & blijver & 4,5 & chiggak \\
\hline lepsy & 2,5 & aflower & 2,5 & lluvio & 5,7 & atwitch & 2,3 & blowgun & 4,5 & chincof \\
\hline rasax & 2,7 & agacant & 2,4 & lquier & 2,3 & aufgabe & 3,6 & ogatyr & 6,7 & hkalik \\
\hline rasax & 5,7 & agalaxy & 2,6 & Iveloz & 5,7 & auksinu & 4,7 & ohorok & 4,7 & hutzpa \\
\hline & 2,5 & gapeti & 2,4 & midoxy & 2,6 & autocab & 4,7 & jjour & 4,6 & \\
\hline sorbs & 2,6 & agapeti & 2,7 & midoxy & 4,6 & & 3,7 & oozify & 4,6 & lawker \\
\hline & 2,6 & agarwal & 2,5 & moving & 2,4 & awapuhi & 2,7 & Ouquet & 3,4 & $\log w y n$ \\
\hline & 2,4 & agarwal & 2,7 & amplify & 2,6 & awapuhi & 5,6 & boxroom & 3,4 & nemial \\
\hline urban & 2,5 & agathin & 2,5 & ampyxes & 2,5 & awfully & 2,3 & breadth & 5,6 & obwebs \\
\hline quia & 2,4 & agavose & 2,4 & mtrack & 2,3 & awkward & 2,3 & breakax & 5,7 & olauxe \\
\hline quia & 4,7 & agelong & 2,7 & amtrack & 2,7 & awnings & 2,6 & risque & 3,5 & luxe \\
\hline known & 2,6 & aggrade & 2,6 & ngareb & 3,7 & axfetch & 2,7 & rusque & 3,5 & \\
\hline natic & 2,3 & aggrege & 2,6 & ngeyok & 5,7 & axif & 2,4 & ryndza & 5,6 & itje \\
\hline nyc & 6,7 & agitato & 2,7 & ngkhak & 3,4 & xmaker & 2,3 & ozoa & 3,5 & itje \\
\hline onyx & 2,7 & aglycon & 2,4 & lorexy & 2,6 & aker & 2,5 & zart & 3,4 & $\operatorname{mixt}$ \\
\hline & 2,6 & agneaux & 2,7 & rtism & 1,2 & nuth & 2,5 & pig & 5,7 & lab \\
\hline tyl & 2,6 & agpaite & 2,3 & arejo & 2,6 & azofier & 2,7 & wig & 3,5 & oba \\
\hline wlut & 2,4 & agraffe & 2,5 & & 1,4 & azoxime & 2,6 & & 4,5 & \\
\hline azzle & 2,4 & agraffe & 2,6 & pojove & 2,4 & & 2,6 & aapeba & 4,6 & owherb \\
\hline izzle & 2,5 & agrapha & 2,5 & quabib & 2,7 & azul & 3,6 & ajuela & 3,5 & rajuru \\
\hline & 2,3 & agrapha & 2,6 & quatic & 2,7 & azumbre & 2,5 & anezou & 5,7 & rivetz \\
\hline vism & 2,4 & agreges & 2,5 & aqueous & 2,5 & azumbre & 2,6 & ataz & 3,7 & aby \\
\hline & 2,3 & aground & 2,5 & arcform & 3,4 & & 4,5 & & 4,7 & \\
\hline & 2,7 & ahur & 3,6 & & 3,4 & & 3, & & 6,7 & \\
\hline & 2,3 & aiku & 3,5 & & 1 , & & 3,7 & oxy & 4,6 & eno \\
\hline & 6,7 & ajan & 2,5 & & 2 , & & 4,6 & & 6,7 & eno \\
\hline & 2,3 & $\mathrm{ca}$ & 2,4 & arvejon & 3,5 & & 1,6 & wba & & \\
\hline & 2,5 & & 2,6 & & 2,6 & & 3,6 & vaa & 5,7 & zed \\
\hline & 3,6 & akazgin & 2,4 & shcake & 3,4 & & 4,6 & awquaw & 3,4 & hesne \\
\hline & 1,3 & akmudar & 2,3 & shfall & 3,4 & batuque & 4,5 & cesser & 1,2 & queen \\
\hline oks & 2,3 & akvavit & 2,3 & shkoko & 3,4 & bawdric & 3,7 & ceilidh & 6,7 & devwsor \\
\hline & 2,6 & akvavit & 2,5 & ashkoko & 4,6 & baycuru & 3,7 & centrev & 1,7 & dghaisa \\
\hline onef & 4,7 & alcayde & 5,6 & ashrafi & 2,6 & bdellid & 1,2 & centrev & 2,7 & direxit \\
\hline
\end{tabular}


7-letter words (continued)

\begin{tabular}{|c|c|c|c|c|c|c|c|c|c|c|}
\hline & 4,7 & everwho & 2,6 & hajilij & 1,7 & jazzbow & 3,7 & lobiped & 3,5 & offbeat \\
\hline disgulf & 4,7 & cverwho & 5,7 & hajilij & 3,7 & jazzbow & 4,5 & lockbox & 1,7 & \\
\hline isrobe & 1,6 & evoking & 2,4 & hajilij & 4,7 & jazzbow & 4,7 & lokaose & 4,5 & offtype \\
\hline jellab & 2,5 & evzones & 2,3 & hajilij & 5,7 & jestful & 1,5 & lulavim & 5,7 & offtype \\
\hline jellab & 2,7 & eweries & 1,2 & hajilij & 6,7 & jiffies & 1,3 & mahaleb & 3,7 & offtype \\
\hline jibbah & 2,4 & exarchy & 2,6 & handjar & 1,5 & jikungu & 6,7 & mahjong & 3,4 & oficina \\
\hline ibbah & 2,7 & excerpt & 2,6 & hazanim & 3,7 & jinjili & 4,7 & lahzors & 3,4 & gtiern \\
\hline ogvane & 3,4 & exergue & 2,5 & hemiamb & 1,7 & jinxing & 1,4 & narkkaa & 6,7 & mnific \\
\hline omajig & 1,5 & exocarp & 2,7 & hexagyn & 3,6 & jivatma & 3,6 & matzahs & 4,6 & onwards \\
\hline omajig & 3,5 & exquire & 2,3 & hexarch & 3,6 & joubarb & 1,7 & mazdoor & 3,4 & oopuhue \\
\hline omajig & 5,7 & exr & 2,3 & hexaxon & 3,5 & yngine & 1,2 & hazeful & 3,5 & ppaqued \\
\hline rawoff & 2,7 & extbo & 2,4 & hijinks & 3,6 & chcha & 4,5 & lolo & 1,2 & aqued \\
\hline & 4,5 & eyebrow & 2,7 & hoaxing & 1,4 & kahawai & 1,3 & & 4,7 & assa \\
\hline & 5,6 & fabliau & 1,7 & homeown & 4,6 & kajawah & 3,7 & mesivta & 5,6 & fgild \\
\hline cdame & 3,4 & fahlerz & 1,7 & ookupu & 6,7 & kankrej & 1,7 & mikrkra & 3,5 & utjazz \\
\hline nbcow & 4,5 & falloff & 3,7 & oosgow & 4,5 & kankrej & 3,7 & mikvahs & 4,6 & utjazz \\
\hline & 3,7 & fars & 6,7 & oosgow & 5,7 & kankrej & 4,7 & minisub & 5,7 & utjin $x$ \\
\hline ivir & 4,5 & feri & 1,6 & hopvine & 3,4 & kankrej & 5,7 & minxish & 4,7 & butjump \\
\hline & 5,6 & & 3,7 & howadji & 1,6 & kankrej & 6,7 & & 3,4 & utswum \\
\hline & 2,6 & fixa & 4,7 & wadji & 3,6 & & 1,4 & & 4,5 & \\
\hline luff & 4,7 & if & 5,7 & owadji & 3,7 & ave & 3,4 & mitzvah & 4,5 & \\
\hline onin & 2,3 & orded & 1,2 & umbuzz & 1,7 & ellegk & 6,7 & mizmaze & 3,4 & verapt \\
\hline & 2,3 & laxman & 4,5 & umbuzz & 3,7 & ethibh & 6,7 & mizrach & 3,4 & verjob \\
\hline & 2,4 & fort & 1,6 & unfysh & 4,5 & & 1,3 & mizrach & 3,6 & oviform \\
\hline & 2,6 & fort & 5,6 & tu & 5,6 & kibbutz & 3,7 & tic & 1,2 & ovipara \\
\hline & 1,5 & fort & 5,6 & utzpah & 4,5 & kickoff & 1,7 & njah & 5,7 & wnself \\
\hline & 2,5 & & 3,5 & droxy & 1,6 & xia & 1 , & low & 3,7 & owtchah \\
\hline & 2,6 & & 4,5 & droxy & 2,6 & & 4, & & 5,7 & oxbrake \\
\hline & 3,6 & & 2,5 & roxy & 3,6 & & 3,6 & & 5,7 & xhuvud \\
\hline & 2,5 & ite & 1,3 & leite & 1,2 & joy & 4 , & & 4 & \\
\hline & 2,4 & en & 3,4 & ithad & 2,5 & kingcob & 4,7 & & 2,4 & \\
\hline like & 2,6 & eitjie & 1,5 & xebana & 1,2 & kinkhab & 4,7 & Iqua & 1,5 & oxyphil \\
\hline sdem & 2,7 & eotaxy & 1,6 & prevu & 2,7 & knowhow & 4,7 & ndoderm & 1,2 & oxyphil \\
\hline & 2,3 & ernitz & 2,7 & prevu & 6,7 & kokowai & 3,5 & doderm & 2,4 & ozonify \\
\hline & 3,7 & & 4,6 & ual & 1,4 & kowbird & 1,3 & mbo & 1,6 & ozotype \\
\hline & 5,7 & & 4,6 & sh & 3,4 & kumquat & 1,4 & pe & 1,6 & ype \\
\hline & 2,3 & & 4,7 & & 5,6 & cvutzah & 2 & & 1,6 & pakchoi \\
\hline & 2,3 & & 2,4 & & 3,6 & & 2, & jic & 6,7 & alazzi \\
\hline & 2,6 & & 6,7 & & 1,4 & enku & 4,5 & newmown & 4,6 & panchax \\
\hline & 1,6 & & 1,6 & & 1,6 & & 1,6 & & 1,6 & \\
\hline & 2,6 & & 5,6 & griv & 1,7 & ork & 3,4 & & 1 & vak \\
\hline & 2,7 & randma & 5,6 & griv & 2,7 & kin & 4,5 & & 2 & \\
\hline & 1, & dpa & 5,6 & ggriv & 3,7 & $\mathrm{ez}$ & 1,7 & & 1, & nx \\
\hline inox & 2,7 & hen & 4,5 & ogriv & 4,7 & laissez & 4,7 & onsync & 5,7 & phloxin \\
\hline & 2,5 & laxima & 1,4 & ogriv & 6,7 & laissez & 5,7 & ync & 6,7 & pickaxe \\
\hline uulei & 2,7 & laxima & 2,4 & toubou & 2,7 & lalaqui & 1,5 & & 1,4 & ter \\
\hline calop & 2,7 & jjerat & 1,3 & vresse & 2,3 & lambeau & 1,7 & ounize & 1,6 & piquing \\
\hline & 2,3 & veduck & 2,6 & resse & 2,4 & lambkin & 4,5 & rarucu & 1,2 & pirojki \\
\hline & 1,5 & & 2,7 & & 2,6 & & 3,4 & ylghau & 2,7 & pirojki \\
\hline & & & 2,3 & & & & 3 , & akmoss & 3,4 & pizzazz \\
\hline & 2 & & 2 & & & & 6 , & & 2,7 & pizzazz \\
\hline & 3 , & & 3 & & & & 5 , & & 1, & pizzazz \\
\hline & 1, & & 3,4 & & 1,3 & & 3,5 & & 4,5 & placebo \\
\hline & 2,4 & hahnium & 1,3 & jaquima & 1,3 & lixivia & 3,5 & odalisk & 2,7 & planxty \\
\hline
\end{tabular}


7-letter words (continued)

\begin{tabular}{|c|c|c|c|c|c|c|c|c|c|c|}
\hline planxty & 4,5 & resojet & 3,5 & sketchy & 2,5 & tariqat & 1,5 & unwiped & 3,5 & xerarch \\
\hline planxty & 5,7 & revamps & 3,6 & sklater & 2,3 & taxiway & 3,5 & unzoned & 1,3 & xeroxed \\
\hline playbox & 4,7 & reviews & 3,6 & skyjack & 2,6 & taxpaid & 3,4 & upclimb & 2,7 & xoanona \\
\hline playoff & 4,6 & rhubarb & 2,7 & skyjack & 3,4 & tezkere & 3,4 & uphhove & 3,4 & xoanona \\
\hline playoff & 4,7 & ribzuba & 4,6 & slapdab & 3,7 & theyaou & 4,7 & upknell & 2,3 & oanona \\
\hline pogonip & 4,7 & rikshaw & 3,7 & slapdab & 4,7 & thruway & 4,5 & upmount & 2,3 & oanona \\
\hline & 4,6 & oomthy & 4,5 & slumgum & 4,5 & hwaite & 2,3 & uppowoc & 5,7 & \\
\hline bolladz & 6,7 & roubouh & 6,7 & slurbow & 3,7 & jenkal & 2,5 & upvomit & 2,3 & \\
\hline ookawn & 4,6 & ryotwar & 1,2 & smalmed & 2,5 & jenkal & 2,7 & urotoxy & 1,6 & \\
\hline dock & 3,4 & afflow & 3,7 & maragd & 6,7 & tmemata & 1,2 & ushabti & 5 & yachter \\
\hline lamu & 6,7 & apajou & 3,5 & mopple & 2,4 & tomjohn & 4,6 & uspoken & 2,5 & yadayim \\
\hline ave & 4,6 & sapajou & 5,7 & navvle & 5,6 & tomjohn & 6,7 & uswards & 2,3 & yaffler \\
\hline & 4,6 & satlijk & 1,6 & ovenez & 5,7 & topazes & 3,5 & & 1,2 & affler \\
\hline & 1,7 & satlijk & 5,6 & preeuw & 5,7 & toronja & 1,6 & bus & 1,3 & guaza \\
\hline pristav & 2,7 & satlijk & 6,7 & spreeuw & 6,7 & totquot & 3,4 & Iwe & 1,6 & \\
\hline ristav & 3,7 & sattvic & 4,5 & squabby & 2,6 & tramway & 4,5 & we & 4,6 & \\
\hline ristav & 4,7 & savelha & 5,6 & quacco & 2,5 & ransfd & 6,7 & we & 5,6 & arwhip \\
\hline ristav & 5,7 & savssat & 3,4 & quacco & 2,7 & rapeze & 4,6 & vandyke & 1,6 & ashmac \\
\hline & 6,7 & awarra & 3,7 & quaddy & 2,6 & triduum & 5,6 & yke & 5,6 & awnups \\
\hline & 6,7 & canmag & 5,7 & squamae & 2,5 & trisulc & 6,7 & & 1,4 & root \\
\hline & 4,5 & scarrow & 2,7 & squiffy & 2,6 & trumeau & 4,7 & & 1,3 & \\
\hline & 4,6 & cavage & 2,4 & squinny & 2,6 & tubifex & 3,7 & & 1,4 & iva \\
\hline & 3,7 & chillu & 2,7 & tambha & 5,6 & & 5,7 & my & 3,6 & \\
\hline imo & 4,7 & chizzo & 4,6 & andby & 2,6 & $e$ & 4,6 & & 5,7 & \\
\hline sm & 4,5 & $\mathrm{pp}$ & 6,7 & & 5, & ism & 2,7 & & 1 , & ker \\
\hline ed & 2,5 & schtoff & 3,7 & tanjen & 2, & sof & 2,7 & & 1 & \\
\hline fer & 2,5 & chwarz & 4,7 & anzas & 2,5 & zaddik & 2,4 & yingly & 1 , & \\
\hline mas & 2,3 & rubby & 2,6 & pgap & 4,5 & dik & 2 & pou & 5,7 & um \\
\hline vyl & 5,6 & ypphi & 3,6 & lavify & 4,6 & tzol & 2,5 & nefou & 5,7 & irum \\
\hline & 2,3 & eamrog & 4,5 & bfief & 4,7 & tzon & 2,5 & hups & 4,6 & ers \\
\hline qabbala & 1,3 & ejeant & 3,5 & bking & 3,4 & & 2,4 & wave & 3,6 & \\
\hline & 1,3 & mifib & 5,7 & boval & 3,5 & st & 1,2 & wav & 3,7 & tvo \\
\hline quahaug & 1,4 & quoia & 3,6 & bplow & 3,7 & ong & 1,2 & sic & 1,7 & \\
\hline qualify & 1,6 & rozem & 5,7 & btext & 1,6 & & 1,5 & & 1,6 & \\
\hline quayage & 1,6 & & 4,7 & & 3,6 & & 2,6 & & 3,6 & \\
\hline & 1,4 & & 2,4 & & 4,7 & xom & 4,5 & & 4,6 & \\
\hline & 1,5 & & 2,3 & & 2,3 & & 5,6 & & 5 , & \\
\hline ui & 1,7 & etz & 3,7 & & 3,7 & ffo & 5,6 & & 5,7 & \\
\hline & 1,6 & negetz & 4,7 & vabite & 2, & own & 3,6 & & 1,5 & \\
\hline quinyie & 1,5 & ohjis & 4,5 & wallow & 2,7 & & 1 , & $\mathrm{am}$ & 5,7 & bies \\
\hline quorums & 1,6 & shravey & 5,7 & swivels & 2,4 & unfrizz & 2,7 & & 4,5 & \\
\hline rajasic & 3,7 & shutoff & 3,7 & swounds & 2,6 & unfrizz & 4,7 & wolfkin & 4,5 & hem \\
\hline redoubt & 6,7 & sjambok & 2,7 & syntagm & 6,7 & ungyved & 4,5 & wongsky & 4,6 & idal \\
\hline reeject & 1,4 & skatiku & 2,7 & tahgook & 3,4 & unkempt & 3,6 & wraxled & 1,4 & zooming \\
\hline reeject & 3,4 & skeezix & 2,5 & tallyho & 5,6 & unplumb & 3,7 & wrongdo & 5,6 & otaxy \\
\hline reequip & 4,7 & skeezix & 2,7 & tallyho & 5,7 & & 3,6 & & 5,6 & zootype \\
\hline reexpel & 1,4 & skeezix & 5,7 & tamandu & 6,7 & unquick & 3,7 & & 1,5 & zwitter \\
\hline reexpel & 4,5 & skegger & 2,4 & tanquam & 4,7 & unvexed & 3,5 & & 1,5 & zymogen \\
\hline repique & 3,5 & skeough & 2,5 & tapasvi & 5,6 & unwaxed & 3,5 & xerarch & 1,6 & zymomin \\
\hline
\end{tabular}

\section{8-letter words}

abatjour $\quad 2,5$ abatjour $\quad 5,8$ abhinaya $\quad 2,7$ abhiseka $\quad 2,7$ abnormal 2,6 abogados 2,4 aboideau $\quad 5,8$

abrachia $\quad 2,6$ abrocome $\quad 2,7$ absfarad $\quad 2,4$ 
8-letter words (continued)

\begin{tabular}{|c|c|c|c|c|c|c|c|c|c|}
\hline abshenry & 2,4 & ajourise & 2,5 & aperitif & 5,8 & babajaga & 5,7 & breloque & 3,6 \\
\hline absinthe & 2,7 & ajourise & 2,7 & apneusis & 2,3 & backveld & 4,5 & bringsel & 5,6 \\
\hline abulyeit & 2,5 & ajutment & 2,4 & apoplexy & 4,7 & baetzner & 5,6 & brinjaul & 5,8 \\
\hline abuseful & 2,6 & ajutment & 2,5 & apoxesis & 2,4 & baiocchi & 5,7 & britzska & 5,6 \\
\hline acervuli & 5,6 & akalimba & 2,7 & applique & 2,6 & ballahou & 3,8 & brouhaha & 5,7 \\
\hline acetoxim & 6,8 & akalimba & 5,7 & aprendiz & 2,8 & ballahou & 4,8 & browache & 4,6 \\
\hline acneform & 2,3 & akamatsu & 2,8 & aprendiz. & 4,8 & balwarra & 4,8 & bryozoum & 5,7 \\
\hline acquaint & 3,7 & akazgine & 2,4 & aprendiz & 7,8 & bandboxy & 3,7 & buckjump & 2,5 \\
\hline acxoyatl & 2,3 & akazgine & 4,5 & aquacade & 2,7 & bandboxy & 4,7 & buckjump & 3,5 \\
\hline adelopod & 2,6 & akmuddar & 2,3 & aquilino & 2,8 & bandboxy & 5,7 & buckjump & 4,5 \\
\hline adenoids & 2,7 & akmuddar & 2,4 & arkansas & 2,3 & banxring & 1,4 & buhlbuhl & 7,8 \\
\hline adequacy & 4,7 & alamiqui & 2,6 & arquebus & 2,3 & barometz & 2,8 & buzzbomb & 3,7 \\
\hline adjutage & 3,7 & alcazava & 5,7 & arrhizal & 4,6 & barometz & 5,8 & buzzbomb & 3,8 \\
\hline adjutrix & 2,8 & aldehyde & 6,7 & asfetida & 2,3 & barukhzy & 6,7 & buzzbomb & 4,8 \\
\hline adjutrix & 3,8 & alefzero & 4,5 & ashberry & 3,4 & bazookas & 3,6 & buzzword & 3,5 \\
\hline admixing & 2,5 & alfaquin & 3,5 & ashstone & 3,4 & becobweb & 5,6 & bywalker & 2,3 \\
\hline adnerval & 2,6 & alforjas & 2,6 & askewgee & 5,6 & bedquilt & 3,4 & cabreuva & 6,7 \\
\hline adsheart & 2,4 & alforjas & 3,6 & asparkle & 2,6 & bedrowse & 6,7 & cachibou & 4,8 \\
\hline affamish & 2,5 & alforjas & 4,6 & asphyxia & 5,6 & befavour & 3,5 & cachibou & 6,8 \\
\hline affamish & 2,8 & alhacena & 2,3 & aspiquee & 2,5 & bejabers & 3,5 & cacomixl & 5,7 \\
\hline affectum & 2,8 & alkermes & 3,6 & aswooned & 2,3 & belduque & 4,6 & cacomixl & 7,8 \\
\hline afforced & 2,6 & alkoxide & 3,5 & asylabia & 3,6 & belduque & 5,6 & cacoxene & 3,5 \\
\hline affright & 2,7 & alkylize & 4,7 & attababy & 2,7 & benjamin & 4,6 & caixinha & 4,7 \\
\hline affydavy & 2,4 & alkyloxy & 2,7 & atwitter & 2,3 & benjamin & 4,8 & caixinha & 6,7 \\
\hline affydavy & 3,4 & alkyloxy & 3,7 & autarkic & 6,8 & benzenyl & 4,7 & calcspar & 4,5 \\
\hline afghanis & 2,3 & alkyloxy & 4,7 & autompne & 6,7 & bequeath & 3,6 & calfkill & 4,5 \\
\hline afghanis & 2,4 & alleluja & 1,7 & avadavat & 2,6 & betocsin & 5,6 & calliope & 5,7 \\
\hline afikomen & 2,3 & alleluja & 2,7 & avellano & 2,8 & betwixen & 4,6 & calzoons & 3,4 \\
\hline afikomen & 2,4 & alleluja & 4,7 & avestruz & 2,8 & bewizard & 3,5 & caprylyl & 5,7 \\
\hline aflicker & 2,6 & almsdeed & 4,5 & avestruz & 3,8 & biajaiba & 4,7 & carboxyl & 4,6 \\
\hline afluking & 2,5 & alquifou & 3,6 & avestruz & 4,8 & biconvex & 4,8 & carpuspi & 6,7 \\
\hline africans & 2,3 & alquifou & 3,8 & aviatrix & 2,8 & biconvex & 6,8 & cavyyard & 4,5 \\
\hline aftereye & 2,7 & alrighty & 2,3 & avifauna & 2,4 & bienvenu & 5,8 & cephalob & 3,8 \\
\hline aftertax & 2,8 & amazeful & 4,6 & awaruite & 2,5 & bijwoner & 3,4 & chaffwax & 5,6 \\
\hline agalaxia & 2,6 & amblyope & 3,7 & awninged & 2,3 & bijwoner & 3,5 & chaology & 3,4 \\
\hline agalloch & 2,7 & amovable & 2,4 & axletree & 2,3 & billywix & 5,8 & cherubim & 6,8 \\
\hline agalloch & 2,8 & amphioxi & 2,7 & axmaking & 2,5 & biquartz & 2,8 & chevesne & 6,7 \\
\hline agedness & 2,4 & amphioxi & 3,7 & axolysis & 2,5 & biquartz & 3,8 & chremzel & 5,6 \\
\hline agendums & 2,5 & amphioxi & 4,7 & azoblack & 2,5 & biquartz & 4,8 & chronaxy & 5,7 \\
\hline aggraded & 2,6 & amphioxi & 5,7 & azoblack & 2,6 & blackgum & 5,6 & churchgo & 6,7 \\
\hline agitprop & 2,8 & amphioxi & 7,8 & azoblack & 2,7 & blauwbok & 4,8 & chutzpah & 3,5 \\
\hline aglimmer & 2,6 & anauxite & 3,5 & azoblack & 2,8 & blauwbok & 5,6 & chutzpah & 5,6 \\
\hline agnizing & 2,5 & anauxite & 4,5 & azoeosin & 2,4 & bloomkin & 5,6 & chutzpah & 6,8 \\
\hline agoniada & 2,7 & angareeb & 3,8 & azoeosin & 2,5 & bluejack & 4,5 & chylific & 3,6 \\
\hline agpaitic & 2,3 & angekkok & 3,5 & azogreen & 2,4 & bombazet & 6,8 & claqueur & 4,7 \\
\hline agraffee & 2,6 & annexive & 5,7 & azohumic & 2,4 & bonnyvis & 5,6 & clavilux & 4,8 \\
\hline agrotype & 2,6 & antefixa & 2,7 & azohumic & 2,8 & bostanji & 1,7 & cliquism & 4,8 \\
\hline agrotype & 2,7 & antefixa & 3,7 & azoimide & 2,4 & bostanji & 3,7 & coadjute & 5,7 \\
\hline aguavina & 2,5 & antefixa & 4,7 & azoimide & 2,5 & bouquets & 3,4 & colloquy & 6,8 \\
\hline aguelike & 2,7 & antefixa & 5,7 & azoimide & 2,7 & bouzouki & 4,8 & comedown & 1,7 \\
\hline ajonjoli & 2,5 & antefixa & 7,8 & azulejos & 2,4 & bowstave & 3,7 & condemns & 6,7 \\
\hline ajonjoli & 2,7 & antifame & 5,7 & azulejos & 2,6 & boxthorn & 3,5 & conelrad & 5,6 \\
\hline ajonjoli & 2,8 & aparejos & 2,6 & azulejos & 3,6 & bracozzo & 4,7 & conferva & 4,7 \\
\hline ajourise & 2,4 & aparejos & 4,6 & azureous & 2,7 & breakaxe & 5,7 & continuo & 7,8 \\
\hline
\end{tabular}


8-letter words (continued)

\begin{tabular}{|c|c|c|c|c|}
\hline corncrib & 4,8 & ecgonine & 2,3 & exequial \\
\hline corvktte & 4,5 & ecmnesia & 2,3 & exfigure \\
\hline cowquake & 3,4 & ecphoria & 2,3 & exonship \\
\hline coxcomby & 3,4 & eddyroot & 2,4 & expounds \\
\hline coxcomby & 3,7 & effluvia & 2,6 & exscribe \\
\hline coxswain & 3,5 & effluxes & 2,6 & extispex \\
\hline crampbit & 5,6 & eggcrate & 3,4 & exuviate \\
\hline crazycat & 4,6 & eggfruit & 2,4 & eyebrows \\
\hline creamcup & 5,8 & egyptian & 2,3 & eyesalve \\
\hline cubbyyew & 5,6 & ejection & 2,7 & eyewater \\
\hline cubbyyew & 6,8 & ejection & 2,8 & fabliaux \\
\hline cubocube & 5,7 & ejective & 2,7 & fahlband \\
\hline cunjevoi & 4,6 & ejicient & 2,3 & farmwife \\
\hline cunjevoi & 4,8 & ejicient & 2,5 & feridjee \\
\hline czarship & 2,5 & ekphoria & 2,3 & feridjee \\
\hline czarship & 2,8 & emajagua & 2,4 & feverfew \\
\hline daiquiri & 4,8 & emajagua & 4,7 & flaxbush \\
\hline dearthfu & 1,8 & emanativ & 1,8 & flaxbush \\
\hline dearthfu & 6,7 & emanativ & 2,8 & flaxdrop \\
\hline dearthfu & 7,8 & emanativ & 3,8 & flaxwife \\
\hline dekagram & 3,5 & emanativ & 4,8 & flicflac \\
\hline demijohn & 3,5 & emanativ & 6,8 & flicflac \\
\hline demijohn & 5,7 & emanativ & 7,8 & flyblown \\
\hline detoxify & 1,5 & embusque & 1,6 & fopperly \\
\hline detoxify & 5,7 & embusque & 2,6 & forzando \\
\hline detraque & 1,6 & embusque & 4,6 & foujdary \\
\hline detraque & 3,6 & emceeing & 2,3 & foujdary \\
\hline devaraja & 1,7 & endrumpf & 1,8 & foujdary \\
\hline devaraja & 2,7 & endrumpf & 6,8 & foxproof \\
\hline devaraja & 3,7 & eneclann & 7,8 & fravashi \\
\hline diapalma & 6,7 & enkolpia & 3,6 & fraxetin \\
\hline diaxonic & 1,4 & enskying & 1,4 & frequent \\
\hline dikaryon & 3,6 & enthalpy & 6,7 & furzetop \\
\hline diptychs & 5,7 & epacmaic & 4,5 & fuzztail \\
\hline disavows & 5,7 & equaeval & 2,6 & fwelling \\
\hline disenjoy & 1,6 & equative & 2,7 & galaxies \\
\hline disjeune & 4,6 & equiaxed & 2,6 & galionji \\
\hline dizzying & 3,5 & equivoke & 2,7 & garookuh \\
\hline djagoong & 2,4 & equuleus & 2,4 & garvanzo \\
\hline djagoong & 2,5 & equuleus & 3,4 & gascromh \\
\hline djasakid & 2,5 & erzahler & 2,3 & gazpacho \\
\hline djasakid & 2,6 & esdragol & 2,3 & gekkonid \\
\hline djasakid & 2,7 & esophagi & 2,8 & geotaxis \\
\hline djasakid & 2,8 & esquisse & 3,7 & ghatwazi \\
\hline dojigger & 2,3 & essexite & 2,5 & ghawazee \\
\hline doorjamb & 5,8 & ethoxide & 2,5 & ghawazee \\
\hline dopchick & 3,4 & euphuize & 5,7 & ghorkhar \\
\hline driftpin & 4,6 & eurythmy & 6,7 & glaistig \\
\hline dummkopf & 4,8 & euxenite & 1,3 & gloxinia \\
\hline dyestuff & 2,7 & evelight & 2,7 & glyoxime \\
\hline dyestuff & 2,8 & eventful & 2,6 & gnatsnap \\
\hline dysorexy & 1,7 & ewelease & 1,2 & golgotha \\
\hline dysorexy & 5,7 & excavate & 2,5 & golkakra \\
\hline earjewel & 4,6 & excysted & 2,4 & granjeno \\
\hline
\end{tabular}

\begin{tabular}{|c|c|c|c|}
\hline granjeno & 5,8 & inparfit & 1,6 \\
\hline guatambu & 1,7 & inquisit & 3,7 \\
\hline guatambu & 6,8 & inscribe & 17 \\
\hline latambu & 7,8 & intonaci & 1,8 \\
\hline ulmohar & 5,6 & inukshuk & 14 \\
\hline guytrash & 2,3 & invoking & 1,5 \\
\hline guzmania & 3,4 & irefully & 1,4 \\
\hline gwerziou & 1,2 & ishpingo & 3,8 \\
\hline gwerziou & 2,8 & isozooid & \\
\hline gymkhana & 3,4 & isozooid & \\
\hline hamingja & 1,7 & iwflower & 2,3 \\
\hline hamingja & 5,7 & iwflower & 2,5 \\
\hline hamingja & 6,7 & iwurthen & 2,3 \\
\hline hanifiya & 5,7 & jacinthe & 1,7 \\
\hline haqueton & 1,3 & jacquard & 4,8 \\
\hline hauynite & 3,4 & jactivus & 1,6 \\
\hline avlagah & 3,4 & jakfruit & 3,4 \\
\hline haznadar & 3,4 & janizary & 1,5 \\
\hline hazzanut & 1,4 & jaquette & 1,3 \\
\hline hecatomb & 1,8 & jarovize & 1,5 \\
\hline hecatomb & 5,8 & jazziest & 1,4 \\
\hline herquein & 1,4 & jeopardy & 1,7 \\
\hline hexandry & 3,6 & jezekite & 1,5 \\
\hline hexylene & 3,4 & jezekite & 3,5 \\
\hline highbrow & 3,8 & jigsawed & 1,6 \\
\hline highjack & 1,5 & jimigaki & 1,8 \\
\hline highjack & 3,5 & jimigaki & 5,7 \\
\hline highjack & 4,5 & jincamas & 1,6 \\
\hline hijacker & 1,3 & jipijapa & 1,5 \\
\hline hijacker & 3,6 & jipijapa & 3,5 \\
\hline himwards & 3,4 & jipijapa & 5,7 \\
\hline hoaxable & 1,4 & jovilabe & 1,7 \\
\hline hogreeve & 1,7 & jovilabe & 3,7 \\
\hline homotaxy & 1,7 & joyproof & 1,8 \\
\hline homotaxy & 3,7 & judaizer & 1,6 \\
\hline hoogaars & 5,6 & juiceful & 1,6 \\
\hline howitzer & 3,6 & kabeljou & 3,6 \\
\hline huajillo & 1,4 & kabeljou & 5,6 \\
\hline hutukhtu & 5,8 & kaivalya & 4,7 \\
\hline hydroxyl & 1,6 & kajugaru & 3,8 \\
\hline hydroxyl & 3,6 & kawakawa & 3,8 \\
\hline ibisbill & 1,2 & kawakawa & 7,8 \\
\hline ibisbill & 2,5 & kazachki & 3,8 \\
\hline identify & 2,7 & kefifrel & 3,5 \\
\hline idiozome & 2,5 & ketazine & 1,5 \\
\hline idleship & 2,8 & ketoxime & 1,5 \\
\hline ijussite & 1,2 & khafajeh & 2,6 \\
\hline & 2,5 & khafajeh & 4,6 \\
\hline ijussite & 2,7 & khafajeh & 6,8 \\
\hline ikeyness & 1,2 & khedival & 1,6 \\
\hline illhumor & 3,4 & kickshaw & 1,8 \\
\hline illiquid & 1,5 & kikawaeo & 5,8 \\
\hline impawned & 2,5 & kinkajou & 4,6 \\
\hline inkmaker & 3,4 & kinkajou & 4,8 \\
\hline
\end{tabular}


8-letter words (continued)

\begin{tabular}{|c|c|c|c|c|c|c|c|c|c|}
\hline kivikivi & 1,7 & mahjongg & 6,8 & myelauxe & 4,7 & onwardly & 1,3 & pidjajap & 6,8 \\
\hline kiwikiwi & 6,7 & mahjongg & 7,8 & myriadth & 6,7 & onyxitis & 1,4 & pincpinc & 4,5 \\
\hline kiwikiwi & 7,8 & mahzorim & 3,4 & mystific & 2,6 & onyxitis & 3,4 & pioupiou & 5,8 \\
\hline kohlrabi & 3,7 & majestic & 3,8 & mystique & 2,6 & ooziness & 1,3 & piquiere & 3,6 \\
\hline komitaji & 5,7 & majorize & 3,7 & nachtmml & 7,8 & oquassas & 1,2 & playdown & 4,7 \\
\hline kommetje & 5,7 & malaxage & 5,7 & namazlik & 1,5 & orabassu & 1,8 & playstow & 4,8 \\
\hline kommetje & 6,7 & malguzar & 5,6 & namazlik & 5,8 & oriflamb & 4,8 & pneumony & 1,2 \\
\hline kugelhof & 1,3 & maneuvre & 6,7 & nastaliq & 1,8 & ossypite & 2,4 & popglove & 3,4 \\
\hline kugelhof & 5,6 & mangabev & 1,8 & nastaliq & 2,8 & ototoxic & 1,6 & popinjay & 3,6 \\
\hline kujawiak & 3,5 & mangabev & 2,8 & nastaliq & 3,8 & outbluff & 4,8 & pourquoi & 5,8 \\
\hline kujawiak & 3,8 & mangabev & 3,8 & nastaliq & 4,8 & outbribe & 1,7 & preenjoy & 3,6 \\
\hline kurumaya & 5,7 & mangabev & 4,8 & nastaliq & 5,8 & outknave & 3,4 & preequip & 3,5 \\
\hline kuvaszok & 5,6 & mangabev & 6,8 & nastaliq & 6,8 & outknave & 4,7 & preequip & 5,8 \\
\hline kuvaszok & 6,8 & mangabev & 7,8 & nastaliq & 7,8 & outquaff & 4,7 & preexact & 5,7 \\
\hline laceleaf & 3,8 & markazes & 4,6 & nepquite & 3,4 & outquaff & 4,8 & prejudge & 3,4 \\
\hline lacqueys & 4,7 & maroquin & 1,5 & nightjar & 1,6 & outswung & 5,6 & projects & 3,4 \\
\hline lambkill & 4,5 & mathemeg & 6,8 & nightjar & 3,6 & outthrob & 4,8 & proverbs & 4,7 \\
\hline landwehr & 5,7 & maximize & 3,7 & nightjar & 4,6 & overgive & 5,7 & proxying & 4,5 \\
\hline landwehr & 6,7 & mazopexy & 3,7 & nightjar & 5,6 & overjade & 5,7 & psykters & 3,4 \\
\hline langlauf & 7,8 & mazourka & 3,5 & nightjar & 6,8 & overpuff & 5,8 & pyengadu & 2,8 \\
\hline lapulapu & 1,8 & melamdim & 5,6 & nonequal & 1,5 & overswim & 6,8 & pyengadu & 7,8 \\
\hline lapulapu & 3,8 & mezuzahs & 5,7 & nonserif & 1,8 & overzeal & 2,5 & pyjamaed & 2,3 \\
\hline lapulapu & 4,7 & mhometer & 1,2 & nonylene & 1,4 & oviducal & 2,6 & qabbalah & 1,3 \\
\hline lapulapu & 7,8 & micromho & 6,7 & noseherb & 1,8 & oxharrow & 2,8 & qadarite & 1,3 \\
\hline lawcraft & 3,7 & micromho & 6,8 & noseherb & 3,8 & oxpecker & 2,6 & qaimaqam & 1,6 \\
\hline lawyerly & 3,4 & midverse & 3,4 & nosotaxy & 1,7 & oxybapha & 2,4 & qaimaqam & 6,7 \\
\hline lazyback & 3,7 & mijnheer & 3,4 & notifyee & 5,6 & oxybapha & 2,7 & qaimaqam & 6,8 \\
\hline lazyship & 3,5 & mijnheer & 3,5 & novatrix & 3,8 & oxybapha & 4,6 & quaggier & 1,5 \\
\hline lazyship & 3,8 & minxship & 1,4 & nowadays & 3,7 & oxyethyl & 2,7 & quartzic & 1,6 \\
\hline leeroway & 1,6 & minxship & 4,6 & nutbrown & 1,7 & oxyurous & 3,4 & quashing & 1,5 \\
\hline leucoryx & 1,8 & miszoned & 3,4 & oblicque & 1,6 & ozarkite & 2,5 & quaylike & 1,7 \\
\hline lherzite & 1,2 & mitzvahs & 4,7 & oblicque & 2,6 & ozobrome & 2,7 & quiaquia & 1,5 \\
\hline libidibi & 5,7 & mnemonic & 1,2 & oblicque & 5,6 & ozokerit & 2,4 & quiaquia & 5,8 \\
\hline limekiln & 7,8 & molybdic & 5,6 & obsolesc & 2,8 & ozokerit & 2,8 & quidnunc & 6,8 \\
\hline limequat & 1,5 & monkeyfy & 6,7 & obtemper & 2,6 & ozophene & 2,5 & quietive & 1,7 \\
\hline lipoxeny & 1,5 & montjoye & 1,5 & obtusish & 2,8 & panicful & 5,6 & quillaja & 1,7 \\
\hline liquamen & 3,6 & mopehawk & 3,7 & octuplex & 2,8 & paradoxy & 1,7 & quillaja & 3,7 \\
\hline liqueurs & 3,6 & moresque & 4,6 & odograph & 2,4 & parhelnm & 6,7 & quillaja & 4,7 \\
\hline lobbygow & 1,8 & motorcab & 6,8 & offbreak & 2,8 & parhelnm & 7,8 & quincunx & 1,8 \\
\hline longjaws & 1,5 & mouseweb & 3,8 & offenses & 2,6 & paroxysm & 5,8 & quincunx & 5,8 \\
\hline longjaws & 4,5 & mozemize & 3,7 & offprint & 2,4 & partykin & 5,6 & quincunx & 6,8 \\
\hline longjaws & 5,7 & mridanga & 1,2 & offprint & 3,4 & payyetan & 3,4 & quinogen & 1,6 \\
\hline lynxlike & 1,4 & mujtahid & 3,4 & offscape & 2,7 & peepshow & 4,8 & quirinca & 1,7 \\
\hline lynxlike & 2,4 & muktatma & 6,7 & offscape & 3,7 & pejorism & 3,8 & quizzify & 4,7 \\
\hline lyophobe & 2,7 & multigap & 6,8 & offwards & 3,4 & penghulu & 4,8 & rahdaree & 3,4 \\
\hline lyophobe & 4,7 & multijet & 4,6 & ofttimes & 2,4 & perijove & 5,7 & raiiform & 3,4 \\
\hline macaasim & 4,5 & multijet & 5,6 & ohmmeter & 1,2 & phorminx & 5,8 & rajaship & 3,8 \\
\hline madrasah & 6,8 & murumuru & 1,8 & ohmmeter & 2,3 & phorminx & 6,8 & rajbansi & 3,4 \\
\hline magdalen & 3,4 & murumuru & 5,8 & oklahoma & 2,7 & photomap & 5,8 & rajbansi & 3,8 \\
\hline magicdom & 5,6 & mustafuz & 1,8 & okolehao & 2,8 & photomap & 6,8 & ramverse & 3,4 \\
\hline maharaja & 1,7 & mustafuz & 2,8 & okshoofd & 2,3 & physique & 3,6 & rchitect & 1,2 \\
\hline maharaja & 3,7 & mustafuz & 3,8 & okshoofd & 7,8 & piblokto & 6,8 & reabsorb & 1,8 \\
\hline mahjongg & 3,4 & mustafuz & 4,8 & okthabah & 2,3 & pidjajap & 4,6 & redivive & 5,7 \\
\hline mahjongg & 4,8 & mustafuz & 6,8 & okthabah & 2,6 & pidjajap & 4,8 & redknees & 3,4 \\
\hline
\end{tabular}


8-letter words (continued)

\begin{tabular}{|c|c|c|c|c|c|c|c|c|}
\hline reenjoin & 5,7 & sgabello & 2,8 & southpaw & 6,8 & szopelka & 2,7 & ubiquity \\
\hline reequipt & 4,7 & shaatnez & 2,8 & sovietic & 3,8 & tacpoint & 3,4 & ugsomely \\
\hline reimbibe & 4,7 & shaatnez & 4,8 & sovkhose & 3,4 & tahkhana & 3,4 & uintaite \\
\hline reinjury & 5,8 & shaatnez. & 6,8 & sovprene & 3,4 & takedown & 3,7 & umbilici \\
\hline remarque & 5,6 & shabbify & 5,7 & sparerib & 2,8 & takrouri & 3,4 & umquhile \\
\hline repondez & 1,8 & shahzadi & 5,8 & sphexide & 2,5 & tallapoi & 6,8 & umquhile \\
\hline repondez & 2,8 & shawabti & 6,7 & spintext & 1,7 & tashreef & 4,8 & umstroke \\
\hline repondez & 3,8 & sheepcot & 5,6 & spintext & 4,7 & tatmjolk & 1,5 & umstroke \\
\hline rerefief & 5,8 & shivzoku & 4,5 & spintext & 7,8 & tatmjolk & 3,5 & umteenth \\
\hline resawyer & 5,6 & shivzoku & 4,8 & spitzkop & 2,5 & tatmjolk & 4,5 & unbelief \\
\hline resqueak & 4,8 & shkotzim & 2,3 & spitzkop & 5,6 & taxiable & 3,6 & unbenumb \\
\hline resqueak & 5,8 & shkotzim & 3,6 & spitzkop & 5,8 & tchincou & 2,8 & uncasque \\
\hline resubmit & 5,6 & shlimazl & 7,8 & spivving & 2,5 & teguexin & 3,6 & underbox \\
\hline retraxit & 6,8 & shouldst & 6,7 & spuilyie & 3,6 & teguexin & 4,6 & underdip \\
\hline revivify & 5,7 & showyard & 4,5 & spuilzie & 5,6 & tendejon & 4,6 & underjaw \\
\hline rglement & 1,2 & shraddha & 5,7 & spytower & 3,6 & tendejon & 6,8 & underjaw \\
\hline rheopexy & 1,7 & sinapize & 5,7 & squailer & 2,5 & textbook & 3,8 & undreamt \\
\hline rhubarby & 3,7 & sitzmark & 4,5 & squamify & 2,7 & tezkirah & 1,3 & unexempt \\
\hline ribazuba & 5,6 & sitzmark & 4,8 & squamify & 5,7 & tezkirah & 3,4 & unexiled \\
\hline rixdaler & 3,4 & skalawag & 2,6 & squshier & 2,4 & thioxene & 2,5 & unexpect \\
\hline roentgen & 5,6 & skatikas & 2,6 & squshier & 2,5 & throwoff & 4,8 & unexuded \\
\hline rollejec & 1,6 & skatoxyl & 2,6 & standoff & 2,8 & thumbkin & 5,6 & unfrizzy \\
\hline rooftops & 4,7 & skatoxyl & 2,7 & stapedez & 2,8 & thumbnut & 5,6 & uninfeft \\
\hline roughhew & 5,6 & skeenyie & 2,6 & stapedez & 4,8 & tieclasp & 7,8 & unjammed \\
\hline roundtop & 5,6 & skewings & 2,7 & stapedez & 5,8 & tjurunga & 2,5 & unjeered \\
\hline rvulsant & 1,2 & skiepper & 2,5 & stayover & 4,6 & tjurunga & 2,6 & unjoyful \\
\hline Sacbrood & 3,4 & skiffled & 2,4 & stickjaw & 1,6 & tjurunga & 2,7 & unopaque \\
\hline samizdat & 5,6 & skijorer & 2,4 & stickjaw & 2,6 & tjurunga & 2,8 & unquayed \\
\hline sandgoby & 5,7 & skindive & 2,7 & stickjaw & 3,6 & tohubohu & 7,8 & unskewed \\
\hline sasanqua & 6,8 & skipjack & 2,5 & stopgaps & 5,7 & toktokje & 1,7 & unswayed \\
\hline scarfpin & 5,6 & skivvies & 2,4 & strength & 6,7 & toktokje & 3,7 & unvizard \\
\hline scherzos & 5,6 & skivvies & 2,5 & stubchen & 4,5 & toktokje & 6,7 & unwaving \\
\hline schiffli & 6,8 & skokiaan & 2,4 & stubchen & 4,6 & topdress & 3,4 & unzipped \\
\hline schiztic & 5,6 & skullcap & 2,6 & subnasal & 3,4 & toxophil & 3,6 & upkindle \\
\hline schmaltz & 2,8 & skycraft & 3,7 & Subtaxer & 3,6 & tranquil & 4,5 & upmaking \\
\hline schmaltz & 3,8 & skylight & 2,6 & subzonal & 3,4 & trapezia & 4,6 & upquiver \\
\hline schmaltz & 4,8 & skylight & 2,7 & sucuruju & 1,7 & tsessebe & 1,7 & upquiver \\
\hline schmaltz & 6,8 & skyscape & 2,7 & sucuruju & 3,7 & tsessebe & 2,7 & upvalley \\
\hline schnapps & 3,7 & skyscape & 3,7 & sucuruju & 4,7 & tukutuku & 3,8 & urfirnis \\
\hline schokker & 2,5 & skywards & 2,7 & sucuruju & 7,8 & turbojet & 4,6 & ushabtiu \\
\hline schryari & 3,5 & slampamp & 5,7 & superadd & 7,8 & turjaite & 1,4 & ushabtiu \\
\hline scofflaw & 2,8 & slangkop & 5,6 & supercow & 3,8 & twelvemo & 5,7 & uturuncu \\
\hline scourway & 4,6 & smaragde & 6,7 & surquidy & 4,7 & twentymo & 2,6 & uxorious \\
\hline screwpod & 5,6 & smokebox & 4,8 & swanskin & 2,6 & twinleaf & 2,8 & vachette \\
\hline scuddawn & 2,7 & smrrebrd & 2,3 & swilltub & 2,8 & twyhynde & 2,4 & vacuumed \\
\hline scuggery & 2,4 & snazzier & 2,4 & swizzler & 2,4 & twyhynde & 2,5 & valiship \\
\hline sedjadeh & 4,8 & sneakbox & 5,8 & swleaves & 2,6 & typikons & 2,5 & vammazsa \\
\hline seldomcy & 6,7 & sneaksby & 5,7 & syllabub & 2,8 & tzedakah & 2,3 & vammazsa \\
\hline sequaces & 3,6 & sneaksby & 6,7 & sylphize & 5,7 & tzedakah & 2,4 & vaqueros \\
\hline sextarii & 3,8 & snuffbox & 5,6 & synacmic & 5,6 & tzedakah & 2,6 & vendaval \\
\hline sforzato & 1,2 & snuffkin & 5,6 & szlachta & 2,3 & tzedakah & 2,7 & venkisen \\
\hline sforzato & 2,5 & socmanry & 3,4 & Szlachta & 2,4 & tzitzith & 2,3 & verjuice \\
\hline sforzato & 2,8 & solonetz & 2,8 & szlachta & 2,5 & tzitzith & 2,5 & vermoulu \\
\hline sgabelli & 2,8 & solonetz & 3,8 & szopelka & 2,6 & ubiquity & 1,2 & vicomtes \\
\hline
\end{tabular}


8-letter words (continued)

$\begin{array}{llllllllll}\text { videruff } & 1,8 & \text { xenocyst } & 1,8 & \text { yardkeep } & 1,5 & \text { yuquilla } & 1,3 & \text { zincking } & 1,5 \\ \text { vizcacha } & 3,4 & \text { xenolith } & 1,8 & \text { yariyari } & 1,5 & \text { yuquilla } & 1,4 & \text { zirconyl } & 1,7 \\ \text { vowelize } & 3,7 & \text { xenophya } & 1,7 & \text { yarovize } & 1,5 & \text { yuquilla } & 2,3 & \text { zizzling } & 1,3 \\ \text { wahconda } & 3,4 & \text { xeransis } & 1,6 & \text { yawmeter } & 1,6 & \text { zabajone } & 1,5 & \text { zodiacal } & 1,6 \\ \text { warratau } & 1,8 & \text { xerantic } & 1,6 & \text { yawshrub } & 1,8 & \text { zaddikim } & 1,6 & \text { zoeaform } & 1,5 \\ \text { wayleave } & 3,7 & \text { xerantic } & 1,8 & \text { yawshrub } & 3,8 & \text { zaibatsu } & 1,8 & \text { zonelike } & 1,7 \\ \text { wheelbox } & 1,8 & \text { xerophil } & 1,8 & \text { ycleping } & 1,2 & \text { zambomba } & 1,7 & \text { zoograft } & 1,7 \\ \text { whipjack } & 1,5 & \text { xiphioid } & 1,8 & \text { ycleping } & 1,5 & \text { zanyship } & 1,4 & \text { zoomorph } & 1,8 \\ \text { whipjack } & 2,5 & \text { xiphuous } & 1,5 & \text { ydriades } & 1,2 & \text { zanyship } & 1,5 & \text { zoopathy } & 1,7 \\ \text { wilcweme } & 4,5 & \text { xylocarp } & 1,8 & \text { yeldrock } & 1,7 & \text { zanyship } & 1,8 & \text { zoospgia } & 5,6 \\ \text { wilcweme } & 5,7 & \text { xyloidin } & 1,6 & \text { yerbales } & 1,4 & \text { zelatrix } & 1,8 & \text { zootoxin } & 1,6 \\ \text { williwaw } & 6,8 & \text { xylorcin } & 1,5 & \text { yielding } & 1,5 & \text { zemstvos } & 1,6 & \text { zootypic } & 1,6 \\ \text { withvine } & 1,5 & \text { xylorcin } & 1,6 & \text { ylahayll } & 1,2 & \text { zemstvos } & 5,6 & \text { zortzico } & 1,5 \\ \text { wlatsome } & 1,2 & \text { xylotile } & 1,7 & \text { ylahayll } & 1,6 & \text { zetacism } & 1,3 & \text { zortzico } & 5,7 \\ \text { womanize } & 1,7 & \text { xylylene } & 1,4 & \text { yoretime } & 1,7 & \text { zikkurat } & 1,3 & \text { zugzwang } & 1,5 \\ \text { woodgrub } & 5,8 & \text { yahrzeit } & 1,5 & \text { yourself } & 1,8 & \text { zikkurat } & 1,4 & \text { zwieback } & 1,2 \\ \text { wowening } & 1,3 & \text { yajenine } & 1,3 & \text { youwards } & 3,4 & \text { zimbabwe } & 1,7 & \text { zwieback } & 1,5 \\ \text { wraxling } & 1,4 & \text { yammadji } & 1,7 & \text { ypsiloid } & 1,2 & \text { zimbabwe } & 5,7 & \text { zygodont } & 1,5 \\ \text { xenelasy } & 1,4 & \text { yammadji } & 6,7 & \text { ysowndir } & 1,2 & \text { zimbabwe } & 6,7 & \text { zymogene } & 1,5 \\ \text { xenocyst } & 1,6 & \text { yanggona } & 1,4 & \text { ysowndir } & 2,4 & \text { zimbabwe } & 7,8 & \text { zymotize } & 1,7\end{array}$

\section{9-letter words}

$\begin{array}{ll}\text { abatjours } & 1,5 \\ \text { abatjours } & 2,5 \\ \text { abbacomes } & 2,7 \\ \text { abbotship } & 2,9 \\ \text { abcoulomb } & 1,9 \\ \text { abcoulomb } & 2,8 \\ \text { abcoulomb } & 2,9 \\ \text { abovedeck } & 2,4 \\ \text { abovedeck } & 2,9 \\ \text { aburabozu } & 2,9 \\ \text { aburabozu } & 6,9 \\ \text { aburabozu } & 7,9 \\ \text { aburabozu } & 8,9 \\ \text { abysmally } & 2,3 \\ \text { acetoxime } & 2,6 \\ \text { acknowing } & 2,3 \\ \text { acneiform } & 2,3 \\ \text { acockbill } & 2,5 \\ \text { acquiesce } & 3,7 \\ \text { acrestaff } & 2,8 \\ \text { adagietto } & 2,9 \\ \text { addendums } & 2,6 \\ \text { adderwort } & 2,6 \\ \text { adeptship } & 2,9 \\ \text { adespoton } & 2,4 \\ \text { adfluxion } & 2,6 \\ \text { adhesives } & 3,7 \\ \text { adjudging } & 2,6 \\ \text { adsignify } & 2,8 \\ \text { advertize } & 2,8 \\ \text { adviceful } & 2,7\end{array}$

$\begin{array}{ll}\text { affabrous } & 2,5 \\ \text { affidavit } & 2,7 \\ \text { affixture } & 2,5 \\ \text { affraying } & 2,6 \\ \text { affreight } & 2,7 \\ \text { affreight } & 2,8 \\ \text { afghanets } & 2,3 \\ \text { aflatoxin } & 2,3 \\ \text { aflatoxin } & 2,6 \\ \text { aflatoxin } & 2,7 \\ \text { afortiori } & 2,9 \\ \text { afterdays } & 2,8 \\ \text { afterhope } & 2,8 \\ \text { afterking } & 2,6 \\ \text { afterlife } & 2,8 \\ \text { afterrake } & 2,8 \\ \text { aftership } & 2,7 \\ \text { afunction } & 2,3 \\ \text { afunction } & 2,4 \\ \text { afwillite } & 1,3 \\ \text { afwillite } & 2,3 \\ \text { agamobium } & 2,6 \\ \text { agentship } & 2,9 \\ \text { aggrading } & 2,6 \\ \text { aggravate } & 2,6 \\ \text { agitatrix } & 2,7 \\ \text { agitatrix } & 2,9 \\ \text { agitpunkt } & 8,9 \\ \text { aglobulia } & 2,5 \\ \text { agminated } & 2,3 \\ \text { agriology } & 2,8\end{array}$

$\begin{array}{ll}\text { agriotype } & 2,7 \\ \text { agriotype } & 2,8 \\ \text { agrodolce } & 2,5 \\ \text { agrologic } & 2,5 \\ \text { agrologic } & 2,7 \\ \text { agromyzid } & 2,7 \\ \text { agueproof } & 2,6 \\ \text { agueproof } & 2,9 \\ \text { aguinaldo } & 2,8 \\ \text { ahartalav } & 1,9 \\ \text { ahartalav } & 2,9 \\ \text { ahartalav } & 3,9 \\ \text { ahartalav } & 4,9 \\ \text { ahartalav } & 5,9 \\ \text { ahartalav } & 6,9 \\ \text { ahartalav } & 7,9 \\ \text { ahartalav } & 8,9 \\ \text { ahluwalia } & 4,5 \\ \text { ahluwalia } & 5,9 \\ \text { akuammine } & 2,6 \\ \text { alambique } & 5,7 \\ \text { alephzero } & 5,6 \\ \text { alexander } & 4,7 \\ \text { algarroba } & 3,8 \\ \text { aljamiado } & 3,8 \\ \text { aljofaina } & 3,5 \\ \text { alkalemia } & 3,7 \\ \text { alkekengi } & 3,5 \\ \text { amenorrho } & 7,8 \\ \text { amidoxime } & 2,6 \\ \text { amidoxime } & 4,6\end{array}$

$\begin{array}{ll}\text { aminoquin } & 2,6 \\ \text { aminoquin } & 3,6 \\ \text { amphioxus } & 5,7 \\ \text { amyotaxia } & 3,7 \\ \text { angelique } & 3,7 \\ \text { angelique } & 4,7 \\ \text { anhydrize } & 4,8 \\ \text { anklejack } & 3,6 \\ \text { anklejack } & 3,9 \\ \text { annexment } & 5,6 \\ \text { antecornu } & 2,9 \\ \text { anthotaxy } & 1,8 \\ \text { anticivic } & 7,9 \\ \text { antiloquy } & 3,7 \\ \text { antizymic } & 5,7 \\ \text { apivorous } & 2,4 \\ \text { apjohnite } & 2,3 \\ \text { apneustic } & 2,3 \\ \text { apodeipna } & 7,8 \\ \text { applejohn } & 6,8 \\ \text { applejohn } & 7,8 \\ \text { applejohn } & 8,9 \\ \text { aquariums } & 2,8 \\ \text { aquascope } & 2,8 \\ \text { aqueously } & 2,5 \\ \text { aquiclude } & 2,8 \\ \text { archdevil } & 3,7 \\ \text { archilowe } & 5,8 \\ \text { archilowe } & 8,9 \\ \text { archknave } & 4,5 \\ \text { arksutite } & 3,5\end{array}$


9-letter words (continued)

\begin{tabular}{|c|c|c|c|c|c|c|}
\hline arquifoux & 3,9 & barrabkie & 6,7 & burkundaz & 8,9 & cliquedom \\
\hline arquifoux & 7,9 & batardeau & 6,9 & buzzingly & 3,7 & clogdogdo \\
\hline arracacha & 5,8 & bauxitite & 2,4 & bywalking & 2,3 & clubhouse \\
\hline arroyuelo & 5,9 & beachcomb & 4,9 & cabilliau & 6,9 & clyfaking \\
\hline arzrunite & 1,3 & beelzebub & 4,5 & cabilliau & 7,9 & coadjutor \\
\hline arzrunite & 2,3 & beelzebub & 5,9 & cabureiba & 4,8 & coadjutor \\
\hline arzrunite & 3,4 & bejabbers & 3,5 & cabureiba & 6,8 & coadjutor \\
\hline arzrunite & 3,5 & bejezebel & 3,5 & caiquejee & 1,7 & coaxingly \\
\hline asafetida & 2,4 & belzebuth & 4,7 & caiquejee & 4,7 & cockneyfy \\
\hline asexually & 4,5 & bemajesty & 5,6 & caiquejee & 5,7 & cockscomb \\
\hline assoilzie & 6,7 & benvenuto & 4,9 & caiquejee & 7,9 & coffeecup \\
\hline astragali & 2,9 & benzoxate & 4,6 & calaththi & 6,8 & cojudices \\
\hline astrakhan & 6,7 & bequirtle & 1,3 & calcipexy & 4,8 & colloquia \\
\hline astrocyte & 2,7 & berkovtsi & 6,7 & calfdozer & 4,5 & commixing \\
\hline astrulabe & 2,8 & besqueeze & 2,4 & canaanite & 4,5 & comtesses \\
\hline atloaxoid & 2,6 & besqueeze & 4,7 & capsizing & 4,6 & concrfsce \\
\hline avizandum & 2,4 & besqueeze & 4,8 & cardiauxe & 3,8 & conjugata \\
\hline avourneen & 2,4 & bethlehem & 6,7 & cardiauxe & 4,8 & conjugial \\
\hline awfulness & 2,3 & bierstube & 1,8 & carfuffle & 4,7 & conjugium \\
\hline awfulness & 2,4 & bierstube & 5,8 & carnaubyl & 7,8 & contractu \\
\hline awikiwiki & 2,9 & bigarreau & 3,9 & carpetweb & 2,9 & convexity \\
\hline awikiwiki & 6,9 & bilboquet & 2,6 & carpetweb & 4,9 & corejoice \\
\hline awkwardly & 2,3 & bilharzic & 7,9 & carryover & 5,7 & coscoroba \\
\hline awunctive & 2,3 & bissextus & 6,8 & carvoepra & 4,7 & cosmolabe \\
\hline awunctive & 2,8 & blackeyes & 5,7 & carvoepra & 7,8 & cowardice \\
\hline axbreaker & 2,7 & blackjack & 2,6 & cataclysm & 7,9 & crabgrass \\
\hline axlesmith & 2,3 & blackjack & 4,6 & catacumba & 6,8 & crossjack \\
\hline azaleamum & 2,6 & blackjack & 5,6 & cattleyak & 7,9 & crossjack \\
\hline azeotropy & 2,9 & bluejoint & 2,5 & caulotaxy & 4,8 & crownment \\
\hline aziethane & 2,4 & bluejoint & 3,5 & chalumeau & 4,9 & cuckquean \\
\hline aziethane & 2,6 & bobbejaan & 4,6 & chaotical & 3,4 & cupflower \\
\hline azimuthal & 2,4 & bobbejaan & 6,8 & chashitsu & 7,9 & currijong \\
\hline azlactone & 2,3 & bookshelf & 8,9 & cheapjack & 2,6 & czarowitz \\
\hline azlactone & 2,4 & bootjacks & 3,5 & cheapjack & 5,6 & czarowitz \\
\hline azlactone & 2,5 & bordereau & 3,9 & chemisorb & 5,9 & czarowitz \\
\hline azobacter & 2,8 & bosjesman & 4,7 & chemotaxy & 4,8 & czarowitz \\
\hline azobacter & 2,9 & bourasque & 4,7 & cheongsam & 6,7 & czarowitz \\
\hline azocyclic & 2,5 & boxholder & 3,4 & chetverik & 5,9 & damoiseau \\
\hline azocyclic & 2,7 & boxkeeper & 3,4 & chibouque & 2,7 & damoiseau \\
\hline azoformic & 2,4 & boxkeeper & 3,7 & chibouque & 5,7 & danoranja \\
\hline azophenyl & 2,8 & brachyure & 6,7 & chibouque & 6,7 & danoranja \\
\hline azorubine & 2,6 & breakover & 5,7 & chickasaw & 5,7 & danoranja \\
\hline azoxazole & 2,8 & breezeful & 5,7 & chiffrobe & 3,8 & danoranja \\
\hline azureness & 2,3 & breezeway & 5,7 & chiffrobe & 4,8 & danoranja \\
\hline azureness & 2,7 & brickkiln & 8,9 & chiffrobe & 5,8 & danoranja \\
\hline azureness & 2,8 & bubblebow & 3,9 & chivalric & 6,7 & dauerlauf \\
\hline azureness & 2,9 & buckskins & 4,6 & chloriamb & 3,9 & dayabhaga \\
\hline bacbakiri & 3,4 & bungalows & 5,8 & choriambi & 8,9 & decidendi \\
\hline backjoint & 4,5 & burkundaz & 1,9 & chuumnapm & 3,4 & defyingly \\
\hline bahuvrihi & 5,8 & burkundaz & 2,9 & chuumnapm & 4,8 & dehnstufe \\
\hline bajarigar & 3,7 & burkundaz & 4,9 & chuumnapm & 8,9 & demihaque \\
\hline barleymow & 6,9 & burkundaz & 5,9 & clampdown & 4,8 & demijambe \\
\hline barleymow & 7,9 & burkundaz & 6,9 & cliffhang & 4,6 & demijambe \\
\hline baronduki & 6,8 & burkundaz & 7,9 & climaxing & 2,6 & demijambe \\
\hline
\end{tabular}


9-letter words (continued)

\begin{tabular}{|c|c|c|c|c|c|c|c|}
\hline dezinkify & 3,6 & edibility & 2,4 & exemplify & 5,8 & furzechat & 4,7 \\
\hline dezinkify & 6,8 & editorial & 2,4 & exequatur & 2,4 & gallycrow & 1,9 \\
\hline dharmsala & 1,2 & effigiate & 2,5 & exoenzyme & 2,7 & garbanzos & 1,7 \\
\hline diazotype & 4,7 & effuviate & 2,5 & exquisite & 2,3 & gaspergou & 7,9 \\
\hline diazotype & 4,8 & efractory & 2,3 & exstrophy & 2,8 & gauzelike & 4,8 \\
\hline dibenzoyl & 6,8 & eggcupful & 2,4 & extrafine & 2,6 & gazehound & 3,5 \\
\hline dicacodyl & 3,8 & eggcupful & 2,5 & extraquiz & 1,9 & gehlenite & 1,3 \\
\hline dihydroxy & 3,8 & eggcupful & 2,6 & extraquiz & 2,9 & gekkonoid & 1,3 \\
\hline dihydroxy & 4,8 & eggcupful & 2,7 & extraquiz & 3,6 & gigahertz & 1,9 \\
\hline dihydroxy & 5,8 & egregious & 2,5 & extraquiz & 3,9 & gitanemuk & 7,9 \\
\hline dihydroxy & 6,8 & egyptians & 2,3 & extraquiz & 5,9 & giveaways & 3,8 \\
\hline dikegrave & 3,5 & eiderdown & 1,8 & extraquiz & 6,9 & gladkaite & 1,5 \\
\hline dimethoxy & 6,8 & ejaculate & 2,5 & extraquiz & 7,9 & gladkaite & 4,5 \\
\hline dimwitted & 3,4 & ejaculate & 2,6 & facsinile & 3,4 & glueyness & 3,5 \\
\hline disadjust & 1,6 & ejaculate & 2,7 & fahlunite & 1,3 & gmelinite & 1,2 \\
\hline disentomh & 3,9 & ejectable & 2,8 & fantasque & 1,7 & goffering & 1,3 \\
\hline disentomb & 5,9 & ejections & 2,6 & fefnicute & 3,4 & golliwogg & 8,9 \\
\hline disfavour & 4.6 & ejections & 2,7 & fevertwig & 3,7 & gonyaulax & 4,9 \\
\hline dishallow & 4,9 & ejections & 2,9 & filmgoing & 4,5 & gordolobo & 8,9 \\
\hline dismayful & 4,7 & ejectment & 2,6 & fizzwater & 3,5 & grassquit & 1,6 \\
\hline disyoking & 4,6 & ejectment & 2,7 & fizzzwater & 4,5 & grassquit & 3,6 \\
\hline dividivis & 3,7 & ejectment & 2,9 & flambeaux & 2,9 & grassquit & 5,6 \\
\hline djalmaite & 2.5 & ejuration & 2,3 & flambeaux & 5,9 & grihastha & 6,8 \\
\hline djellabah & 2,5 & ejuration & 2,4 & flashbulb & 1,9 & griquaite & 1,4 \\
\hline djellabah & 2.8 & ejuration & 2,5 & flashbulb & 2,9 & groundhog & 6,7 \\
\hline djellabah & 2,9 & ejuration & 2,6 & flashbulb & 4,9 & groundnut & 6,7 \\
\hline djellabah & 7,9 & ejuration & 2,8 & flashbulb & 8,9 & groundsel & 6,7 \\
\hline domeykite & 5,6 & ejuration & 2,9 & flaxboard & 4,5 & grudgekin & 5,7 \\
\hline doorknobs & 5,8 & ekphorize & 2,8 & flecnodal & 4,5 & guarapucu & 6,9 \\
\hline doorknobs & 6,8 & embryulci & 5,9 & floorshow & 4,9 & guarapucu & 8,9 \\
\hline doubleyou & 6,9 & emeership & 2,9 & flummoxed & 1,7 & guberniya & 7,8 \\
\hline doubleyou & 7,9 & enchequer & 2,6 & flummoxed & 3,7 & guejarite & 1,4 \\
\hline douzepers & 4,6 & enhancive & 3,8 & flunkyize & 5,8 & haloesque & 1,7 \\
\hline dovehouse & 3,5 & enjeopard & 3,5 & fluorenyl & 3,8 & hammerkop & 7,9 \\
\hline downcurve & 3,8 & enjeopard & 3,6 & fluxgraph & 4,5 & handyblow & 1,9 \\
\hline downgyved & 6,7 & enterauxe & 1,8 & fluxgraph & 4,8 & handygrip & 6,9 \\
\hline driveaway & 4,7 & entredeux & 6,9 & fluxmeter & 4,5 & harlequin & 1,6 \\
\hline dubbeltje & 2,8 & envyingly & 3,4 & flyleaves & 3,7 & harnessry & 7,8 \\
\hline dubbeltje & 3,8 & eosinlike & 1,2 & folcgemot & 4,5 & harquebus & 1,4 \\
\hline dubbeltje & 4,8 & equiaxial & 2,6 & fopdoodle & 3,4 & hausfraus & 4,5 \\
\hline dubbeltje & 5,8 & equipluve & 7,8 & foreadapt & 6,8 & hazardful & 3,7 \\
\hline dubbeltje & 6,8 & equipping & 2,6 & forejudge & 1,5 & hazardize & 3,8 \\
\hline dubbeltje & 7,8 & equisetic & 2,9 & formoxime & 1,6 & heavyback & 4,5 \\
\hline dubbeltje & 8,9 & equivoque & 1,7 & fortuuned & 5,6 & hebdomcad & 6,7 \\
\hline dumbfound & 4,5 & equivoque & 2,7 & foujdarry & 4,5 & hexadecyl & 3,8 \\
\hline dykereeve & 2,3 & equivoque & 5,7 & foujdarry & 4,8 & hgmotine & 1,2 \\
\hline dziggetai & 1,2 & equoidean & 2,4 & fourquine & 1,5 & hijacking & 1,3 \\
\hline dziggetai & 2,4 & equoidean & 2,5 & frailejon & 1,7 & hijacking & 3,6 \\
\hline dziggetai & 2,5 & escapeway & 2,7 & frailejon & 2,7 & hippocerf & 3,9 \\
\hline dziggetai & 2,9 & etiquette & 2,4 & frailejon & 3,7 & hoaxproof & 1,4 \\
\hline ebrillade & 2,8 & eutaxitic & 1,5 & frijolito & 4,9 & hoaxproof & 4,9 \\
\hline echograph & 3,8 & evilproof & 1,9 & frithborh & 8,9 & holishkes & 6,7 \\
\hline echoizing & 4,6 & executrix & 2,9 & frogskins & 4,6 & homegrown & 1,8 \\
\hline edgemaker & 2,7 & exemplify & 2,8 & frolicful & 6,7 & hornthumb & 4,9 \\
\hline
\end{tabular}


9-letter words (continued)

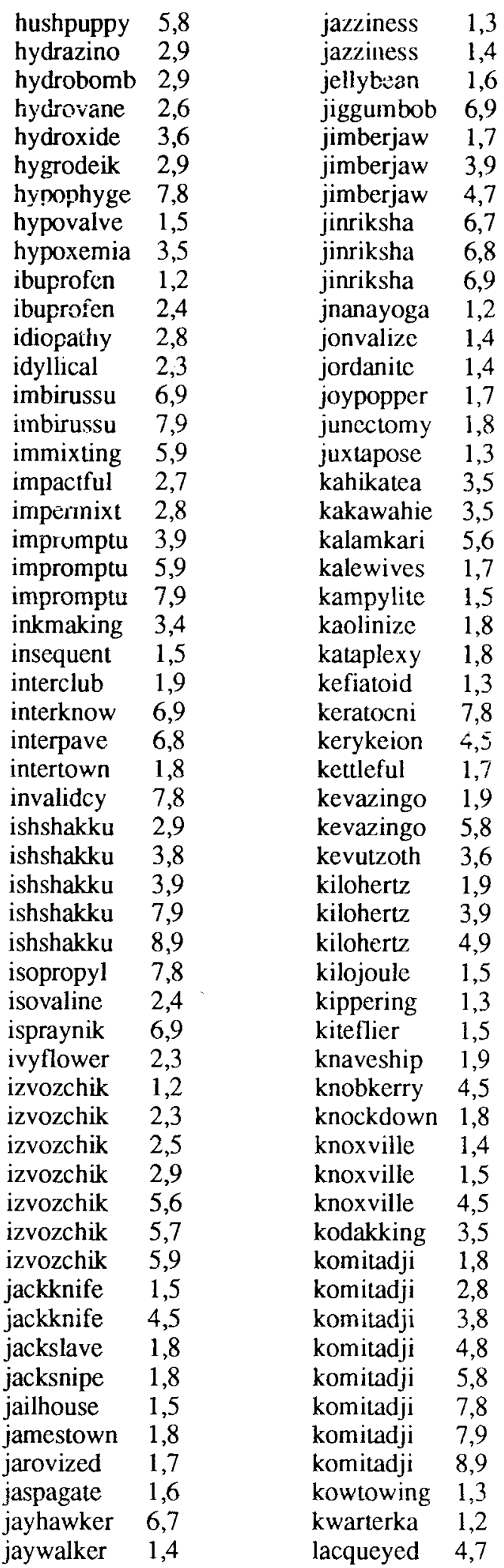

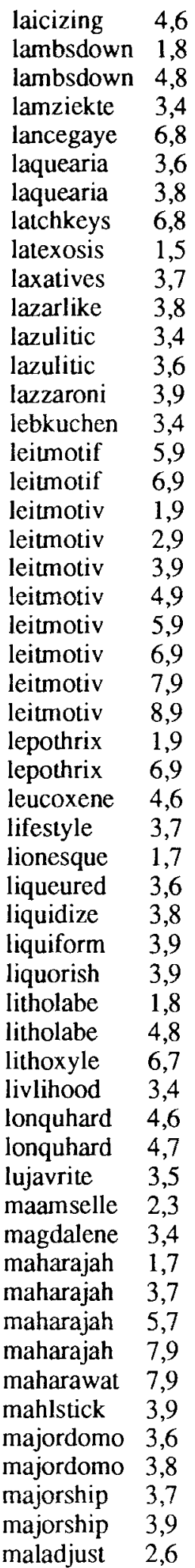

maladjust $\quad 3,6$

malaxator $\quad 5,9$

malikadna 7,8

malikzadi $\quad 5,6$

mandacaru $\quad 6,9$

mandacaru 7,9

mandacaru $\quad 8,9$

mandragun 7,8

mandragvn 8,9

mannequin 1,6

maquereau 3,9

masskanne 3,5

mastopexy $\quad 1,8$

mastopexy $\quad 3,8$

matajuelo 3,5

matajuelo $\quad 5,7$

mawkishly 1,3

mayorship 3,9

mazopathy 3,8

mechitzah $\quad 7,9$

mediatrix $\quad 3,9$

megahertz $\quad 1,9$

megajoule 3,5

megaweber 3,5

mesoxalyl $\quad 5,8$

methoxide 1,6

mezcaline 3,4

midewiwin 6,7

mijnheerl 3,4

minxishly 1,4

minxishly 3,4

miszoning 3,4

mlechchha 1,2

mlechchha 6,8

mlechchha 7,8

mollyhawk 5,8

moneybags 5,8

moneybags 6,8

moneygrub 1,9

monocoque 1,7

monocoque 5,7

monogamou 5,9

monogamou 7,9

mundunugu 6,9

mundunugu 8,9

mustafina $\quad 6,9$

mutawalli $\quad 5,9$

mutesarif 7,9

myxamoeba 3,8

myzostome 3,8

nagkassar $\quad 3,4$

napkining 3,4

naysaying 1,3

nazdrowie 3,4 
9-letter words (continued)

\begin{tabular}{|c|c|c|c|c|c|c|}
\hline nazdrowie & 3,7 & otuingkar & 7,8 & perejonet & 5,7 & preverify \\
\hline nazifying & 3,5 & outbraved & 4,7 & pervenche & 4,8 & princekin \\
\hline nearaways & 1,8 & outdazzle & 4,7 & pezograph & 3,8 & prisiadka \\
\hline neurokyme & 6,8 & outquoted & 1,4 & pharynxes & 5.7 & proexpert \\
\hline neuromyic & 7,9 & outquoted & 3,4 & phenakism & 6,9 & proliquor \\
\hline nightclub & 1,9 & outskirts & 1,5 & phillippi & 6,8 & propinque \\
\hline nihilitic & 1,3 & ovalbumin & 5,7 & phototaxy & 3,8 & proxyship \\
\hline nonglazed & 4,7 & ovaliform & 2,6 & physiqued & 2,6 & pseudopod \\
\hline noninjury & 1,6 & overadom & 2,6 & physiqued & 3.6 & pseudovum \\
\hline noninjury & 3,6 & overbribe & 5,8 & physiurgy & 5,6 & puzzleman \\
\hline nonlawyer & 6,7 & overclimb & 5,9 & piazzetta & 5,9 & pyrazolyl \\
\hline nonliquid & 1,6 & overclimb & 7,9 & pickaxing & 4,6 & quadrivia \\
\hline nonunique & 4,7 & overcrowd & 8,9 & pigeonpox & 3,9 & quaggiest \\
\hline nonwaxing & 4,6 & overdoubt & 5,8 & pigeonpox & 7,9 & quartinho \\
\hline nototribe & 1,8 & overequal & 6,8 & pindjajap & 1,7 & quatrayle \\
\hline noxiously & 1,3 & overequal & 6,9 & pindjajap & 3,5 & quattrini \\
\hline oakenshaw & 3,9 & overequip & 6,9 & pindjajap & 3,7 & quaysider \\
\hline oaktongue & 3,8 & overexert & 1,6 & pindjajap & 4,7 & queenship \\
\hline obeyingly & 2,4 & overexert & 2,6 & pindjajap & 5,7 & quibbling \\
\hline objectize & 3,8 & overfavor & 5,7 & pindjajap & 5,8 & quickside \\
\hline objicient & 3,4 & overheavy & 8,9 & pindjajap & 5,9 & quillajic \\
\hline oblivious & 2,5 & overjawed & 5,7 & pindjajap & 6,9 & quillajic \\
\hline oblongata & 2,9 & overplumb & 5,9 & pindjajap & 7,9 & quillajic \\
\hline oblongish & 2,9 & overquick & 5,8 & pipikaula & 3,5 & quillajic \\
\hline obstruxit & 2,7 & overquick & 5,9 & piquantly & 1,3 & quinaldyl \\
\hline obstruxit & 7,9 & overquick & 6,9 & pituicyte & 4,7 & quinisext \\
\hline odalisque & 2,7 & overskirt & 2,6 & plaintext & 3,8 & quinisext \\
\hline odalwoman & 2,7 & overviews & 5,8 & planetkin & 6,7 & quinisext \\
\hline odorously & 2,6 & overviews & 6,8 & plexiform & 4,6 & quinisext \\
\hline odorproof & 2,9 & ovibovine & 2,4 & plexippus & 4,6 & quinology \\
\hline offerable & 2,7 & ovivorous & 2,4 & plexippus & 4,7 & quinquino \\
\hline offhanded & 3,4 & ovomucoid & 2,4 & podzolize & 3,4 & quinzieme \\
\hline offspring & 2,5 & ownwayish & 2,6 & poetesque & 3,7 & quisutsch \\
\hline oftwhiles & 2,4 & owyheeite & 2,3 & poetiised & 5,6 & quizzical \\
\hline oftwhiles & 2,5 & owyheeite & 2,4 & pohickory & 1,3 & rageproof \\
\hline ogreishly & 1,2 & oxozonide & 2,4 & pokerface & 3,6 & rakesteel \\
\hline okoniosis & 2,7 & oxyketone & 2,4 & poleaxing & 4,6 & rauwolfia \\
\hline okupukupu & 1,9 & oxyketone & 3,4 & polychsia & 6,7 & razorable \\
\hline okupukupu & 2,6 & oxyrhynch & 2,6 & polymyxin & 4,7 & razorback \\
\hline okupukupu & 2,9 & oxyrhynch & 6,8 & polyptych & 7,8 & razoredge \\
\hline okupukupu & 5,8 & oxysulfid & 3,7 & pontianak & 6,9 & razorfish \\
\hline okupukupu & 6,9 & oxytoluic & 7,9 & popgunner & 3,4 & razzberry \\
\hline okupukupu & 8,9 & ozokerite & 2,4 & possumhaw & 5,9 & reconquer \\
\hline omnifidel & 5,7 & ozostomia & 2,6 & possumhaw & 6,9 & rectopexy \\
\hline onwaiting & 1,3 & ozostomia & 2,9 & postulnar & 6,7 & redevelop \\
\hline opalesque & 2,7 & packaging & 4,6 & powderize & 3,8 & redisturb \\
\hline ophthalmy & 7,8 & paeounlae & 6,7 & prayerful & 4,7 & redivulge \\
\hline organonyn & 8,9 & paparazzi & 7,9 & preabsorb & 1,9 & redjacket \\
\hline orthodoxy & 1,8 & paparazzi & 8,9 & preabsorb & 4,9 & reexecute \\
\hline orthodoxy & 2,8 & pasquilic & 4,9 & preabsorb & 5,9 & reexhibit \\
\hline orthodoxy & 6,8 & passersby & 7,8 & preexpect & 5,8 & reforgive \\
\hline orvietite & 2,3 & paynimrie & 6,7 & prehallux & 4,9 & reforsake \\
\hline otherways & 3,8 & peepshows & 2,8 & presubdue & 6,7 & rehydrate \\
\hline ottingkar & 6,7 & peepshows & 4,8 & presubmit & 6,7 & religieux \\
\hline
\end{tabular}


9-letter words (continued)

\begin{tabular}{|c|c|c|}
\hline reliquefy & 5,8 & screwpile \\
\hline renownful & 5,7 & screwship \\
\hline renownful & 5,8 & scrimshaw \\
\hline repiquing & 3,5 & scrimshaw \\
\hline resazurin & 1,5 & scruffman \\
\hline reseizure & 6,7 & semibejan \\
\hline revokable & 3,5 & semibifid \\
\hline rheotaxis & 1,7 & semimajor \\
\hline rheumatiz & 1,9 & semisixth \\
\hline rheumatiz & 2,9 & servitrix \\
\hline rheumatiz & 3,9 & sexangled \\
\hline rheumatiz & 4,9 & sextactic \\
\hline rheumatiz & 5,9 & sextuplex \\
\hline rheumatiz & 6,9 & sexualize \\
\hline rheumatiz & 7,9 & sforzando \\
\hline rhinobyon & 6,7 & sforzando \\
\hline rhizotaxy & 4,8 & sforzando \\
\hline rigamajig & 1,7 & sgraffiti \\
\hline rigamajig & 3,7 & sgraffiti \\
\hline rigamajig & 7,9 & shadowbox \\
\hline ritziness & 3,4 & shahzadah \\
\hline rolleyway & 1,7 & shahzadah \\
\hline roomthily & 4,5 & shakedown \\
\hline roughdraw & 4,9 & sheafripe \\
\hline roughhewn & 1,8 & sheaveman \\
\hline rytidosis & 1,2 & sheepgate \\
\hline safflower & 3,7 & sheepkill \\
\hline salubrify & 5,8 & shelfback \\
\hline salzfelle & 4,5 & sheriffcy \\
\hline sambaquis & 3,6 & shrrinkng \\
\hline sanjakbeg & 6,7 & shrrinkng \\
\hline sapotilha & 7,8 & singkamas \\
\hline sbodikins & 1,2 & siwashing \\
\hline sbodikins & 2,6 & sixteenmo \\
\hline scagliola & 2,4 & skaalpund \\
\hline scampavia & 2,7 & skaalpund \\
\hline scampavia & 5,7 & skaldship \\
\hline scarebabe & 2,8 & skedaddle \\
\hline scarfskin & 5,7 & skeezicks \\
\hline scazontic & 2,4 & sketchpad \\
\hline schemeful & 5,7 & sketchpad \\
\hline scherzoso & 3,9 & sketiotai \\
\hline schizaxon & 5,7 & skewwhiff \\
\hline schizopod & 5,7 & skewwhiff \\
\hline schlemihl & 8,9 & skewwhiff \\
\hline schlimazl & 8,9 & skibslast \\
\hline schmaltzy & 7,8 & skidproof \\
\hline schmaltzy & 8,9 & skilfully \\
\hline schmoozed & 4,7 & skilfully \\
\hline schnauzer & 6,7 & skimmings \\
\hline schweizer & 4,7 & skindiver \\
\hline scoleryng & 7,9 & skinniest \\
\hline scramasax & 2,9 & skintight \\
\hline screaking & 2,6 & skintight \\
\hline
\end{tabular}

$\begin{array}{ll}\text { skiophyte } & 2,7 \\ \text { skipbrain } & 2,9 \\ \text { skipjacks } & 1,5 \\ \text { skipjacks } & 2,5 \\ \text { skipjacks } & 4,5 \\ \text { skullissh } & 2,6 \\ \text { skunkweed } & 2,6 \\ \text { skydiving } & 2,6 \\ \text { skydiving } & 3,6 \\ \text { skyjacker } & 3,4 \\ \text { skylights } & 2,6 \\ \text { skylining } & 2,6 \\ \text { skyscrape } & 2,4 \\ \text { skywriter } & 2,5 \\ \text { sleuthdog } & 7,9 \\ \text { slivovitz } & 1,9 \\ \text { slivovitz } & 2,9 \\ \text { slivovitz } & 3,9 \\ \text { slivovitz } & 4,9 \\ \text { slivovitz } & 6,9 \\ \text { smokejack } & 4,6 \\ \text { smoothify } & 2,8 \\ \text { smutproof } & 2,9 \\ \text { snarleyow } & 7,9 \\ \text { snazziest } & 2,4 \\ \text { snowscape } & 4,6 \\ \text { snowscape } & 4,8 \\ \text { soapsudsy } & 7,8 \\ \text { sobriquet } & 3,6 \\ \text { solemnize } & 5,8 \\ \text { solidillu } & 3,9 \\ \text { solidillu } & 5,9 \\ \text { solidillu } & 7,9 \\ \text { solidillu } & 8,9 \\ \text { solvaated } & 5,6 \\ \text { soumarque } & 6,7 \\ \text { spareribs } & 2,8 \\ \text { spelldown } & 2,8 \\ \text { spiderweb } & 2,9 \\ \text { spiderweb } & 4,9 \\ \text { spiffiest } & 2,4 \\ \text { spillover } & 2,7 \\ \text { splenauxe } & 1,8 \\ \text { splenauxe } & 2,8 \\ \text { spoilfive } & 6,8 \\ \text { springbok } & 6,9 \\ \text { squarecap } & 2,9 \\ \text { stannoxyuake } & 5,8 \\ \text { squibster } & 2,6 \\ \text { squiffier } & 2,5 \\ \text { squshiest } & 2,4 \\ \text { squiest } & 2,5 \\ & 2,7 \\ & \end{array}$

\begin{tabular}{|c|c|}
\hline statesboy & 6,7 \\
\hline statorhab & 2,9 \\
\hline statorhab & 7,9 \\
\hline statorhab & 8,9 \\
\hline steamship & 5,9 \\
\hline steepdown & 2,8 \\
\hline stopovers & 2,6 \\
\hline stratojet & 2,7 \\
\hline stratojet & 3,7 \\
\hline stratojet & 5,7 \\
\hline stratojet & 6,7 \\
\hline stratojet & 7,9 \\
\hline strawyard & 5,6 \\
\hline strayaway & 5,7 \\
\hline streamlet & 6,7 \\
\hline streamway & 6,7 \\
\hline strifeful & 5,7 \\
\hline strongbox & 2,9 \\
\hline strongbox & 5,9 \\
\hline strongbox & 6,9 \\
\hline subaquean & 3,5 \\
\hline subclavii & 7,9 \\
\hline subclique & 4,7 \\
\hline subconvex & 7,9 \\
\hline subluxate & 3,6 \\
\hline subsesqui & 7,9 \\
\hline subsesqui & 8,9 \\
\hline subzonary & 3,4 \\
\hline suffrutex & 3,9 \\
\hline suffrutex & 4,9 \\
\hline sulphamyl & 7,8 \\
\hline sulphoxid & 5,7 \\
\hline sunsquall & 3,5 \\
\hline superbomb & 3,9 \\
\hline superdebt & 6,8 \\
\hline supervive & 6,8 \\
\hline surfrappe & 4,8 \\
\hline swansdown & 2,5 \\
\hline swansdown & 2,6 \\
\hline swarajism & 2,9 \\
\hline swarajism & 6,9 \\
\hline swartzite & 2,6 \\
\hline sweetleaf & 2,9 \\
\hline sweetsome & 2,8 \\
\hline swervable & 2,5 \\
\hline swinepipe & 2,8 \\
\hline swingeour & 2,8 \\
\hline swiveleye & 2,8 \\
\hline syllabify & 2,8 \\
\hline syllabify & 6,8 \\
\hline syncopize & 6,8 \\
\hline syzygetic & 2,4 \\
\hline lacmahack & 3,4 \\
\hline talbotype & 4,8 \\
\hline
\end{tabular}


9-letter words (continued)

\begin{tabular}{|c|c|c|c|c|c|}
\hline tamanowus & 7,8 & underyoke & 6,8 & wapperjaw & 4,7 \\
\hline tariffize & 6,8 & uncxpired & 4,7 & waxflower & 3,7 \\
\hline tasajillo & 1,5 & unextreme & 4,8 & waxworker & 3,7 \\
\hline technique & 3,7 & unfakable & 3,5 & wayfellow & 3,9 \\
\hline technique & 4,7 & unfrizzly & 3,6 & wellqueme & 1,5 \\
\hline teknonymy & 3,7 & unírizzly & 6,9 & wellqueme & 4,5 \\
\hline telakucha & 5,8 & unfrizzly & 7,9 & wellqueme & 5,8 \\
\hline terahertz & 1,9 & unfulsome & 3,8 & wetherteg & 7,9 \\
\hline thiocyano & 6,9 & ungivable & 3,5 & whillywha & 7,8 \\
\hline thriftbox & 5,9 & uniaxally & 3,5 & whillywha & 7,9 \\
\hline throwdown & 5,8 & unimbowed & 4,7 & whirlpuff & 5,9 \\
\hline thumbmark & 5,6 & unjeering & 3,5 & whirlpuff & 6,9 \\
\hline thwartsaw & 4,9 & unjuridic & 3,9 & whitecomb & 1,9 \\
\hline tibourbou & 7,9 & unknocked & 3,6 & whitevein & 1,6 \\
\hline tipstaffs & 6,8 & unluckful & 6,7 & withsayer & 1,7 \\
\hline tonalmatl & 8,9 & unmummify & 6,8 & wlonkhede & 1,2 \\
\hline tonguetip & 5,9 & unmuzzled & 3,5 & wofulness & 1,4 \\
\hline toothcomb & 3,9 & unquadded & 3,7 & wolfhound & 4,5 \\
\hline topazfels & 5,6 & unquaffed & 3,7 & worriecow & 7,9 \\
\hline toxiphagi & 3,9 & unquaking & 3,6 & wouhleche & 3,4 \\
\hline tradeoffs & 6,8 & unquashed & 3,7 & wrongwise & 5,6 \\
\hline trajected & 3,4 & unquizzed & 3,6 & xanthuria & 1,6 \\
\hline trefgordd & 4,5 & unquizzed & 3,7 & xenagogue & 1,4 \\
\hline triptyque & 6,7 & unremoved & 5,7 & xenelasia & 1,4 \\
\hline trousseau & 3,9 & unsexlike & 5,8 & xenelasia & 1,5 \\
\hline trousseau & 5,9 & unskaithd & 8,9 & xenoblast & 1,5 \\
\hline tsktsking & 2,3 & unwavered & 3,5 & xenodochy & 1,8 \\
\hline tunnelway & 6,7 & unzipping & 3,6 & xenograft & 1,8 \\
\hline twifallow & 2,9 & updraught & 2,3 & xerically & 1,7 \\
\hline twigwithy & 2,5 & upflicker & 2,3 & xeromyron & 1,8 \\
\hline twigwithy & 2,8 & upgrading & 2,3 & xerophyte & 1,8 \\
\hline twigwithy & 5,8 & urogomphi & 1,9 & xerostoma & 1,5 \\
\hline twistical & 2,7 & ushership & 2,9 & xerostoma & 1,6 \\
\hline twizzened & 2,4 & uteropexy & 1,8 & xiphistna & 7,8 \\
\hline twodecker & 2,7 & uteropexy & 2,8 & xiphoidal & 1,9 \\
\hline tzaddikim & 2,5 & vajrasana & 1,3 & xiphosure & 1,7 \\
\hline tzaddikim & 2,6 & vajrasana & 3,4 & xylindein & 1,5 \\
\hline tzaddikim & 2,7 & vasospasm & 1,6 & xylomancy & 1,8 \\
\hline tzaddikim & 7,9 & vasotribe & 1,8 & xylometer & 1,9 \\
\hline tzarevich & 2,6 & venanzite & 1,6 & yakattalo & 1,3 \\
\hline ubiquious & 2,4 & verjuiced & 4,7 & yakattalo & 1,9 \\
\hline udderlike & 2,8 & vierkleur & 1,5 & yakattalo & 3,9 \\
\hline ugrianize & 2,8 & vitapathy & 1,8 & yamaskite & 1,6 \\
\hline uhtensang & 1,2 & voltivity & 1,6 & yamstchik & 1,6 \\
\hline ulrichite & 2,3 & vorondreo & 8,9 & yamstchik & 1,7 \\
\hline unabjured & 4,5 & vraicking & 1,2 & yarovized & 1,4 \\
\hline unaffixed & 4,7 & waggonway & 5,7 & yarovized & 1,5 \\
\hline unantique & 1,7 & waggonway & 6,7 & yarovized & 1,7 \\
\hline unawfully & 4,6 & wakefully & 3,6 & yawnfully & 1,5 \\
\hline unbedewed & 3,7 & wakerobin & 3,7 & yawnfully & 1,6 \\
\hline unbeknown & 3,8 & wakizashi & 1,9 & yawnproof & 1,5 \\
\hline unbrutify & 3,8 & wakizashi & 3,5 & yawnproof & 1,9 \\
\hline underjoin & 3,6 & wapperjaw & 1,7 & yearnings & 1,8 \\
\hline underrobe & 1,8 & wapperjaw & 3,7 & yellowcup & 1,7 \\
\hline
\end{tabular}

$\begin{array}{ll}\text { yellowcup } & 6,7 \\ \text { yellowfin } & 1,7 \\ \text { yeomaness } & 1,3 \\ \text { ycomaness } & 1,5 \\ \text { yeshivahs } & 1,8 \\ \text { yeshivahs } & 6,8 \\ \text { yesterday } & 1,7 \\ \text { yestereve } & 1,8 \\ \text { yieldable } & 1,7 \\ \text { yiverness } & 1,3 \\ \text { yodelling } & 1,3 \\ \text { yodelling } & 1,5 \\ \text { yohimbine } & 1,3 \\ \text { yohimbine } & 1,4 \\ \text { yohimbine } & 1,5 \\ \text { youngster } & 1,9 \\ \text { youthsome } & 1,8 \\ \text { youthtide } & 1,8 \\ \text { youthwort } & 1,8 \\ \text { ytterbium } & 1,9 \\ \text { yuleblock } & 1,5 \\ \text { yuquillas } & 1,3 \\ \text { yuquillas } & 1,4 \\ \text { yuquillas } & 2,3 \\ \text { zarabanda } & 1,5 \\ \text { zarzuelas } & 4,5 \\ \text { zealproof } & 1,9 \\ \text { zebralike } & 1,8 \\ \text { zemimdari } & 5,6 \\ \text { zendikite } & 1,6 \\ \text { zermahbub } & 1,9 \\ \text { zermahbub } & 5,9 \\ \text { zeroaxial } & 1,6 \\ \text { zestfully } & 1,5 \\ \text { zitherist } & 1,3 \\ \text { zoiatrics } & 1,3 \\ \text { zoocystic } & 1,6 \\ \text { zoofulvin } & 1,7 \\ \text { zoothecia } & 1,7 \\ \text { zuurveldı } & 1,5 \\ \text { zuurveldı } & 8,9 \\ \text { zwanziger } & 1,2 \\ \text { zwanziger } & 1,5 \\ \text { zwanziger } & 5,7 \\ \text { zygadenin } & 1,5 \\ \text { zymogenic } & 1,5\end{array}$

